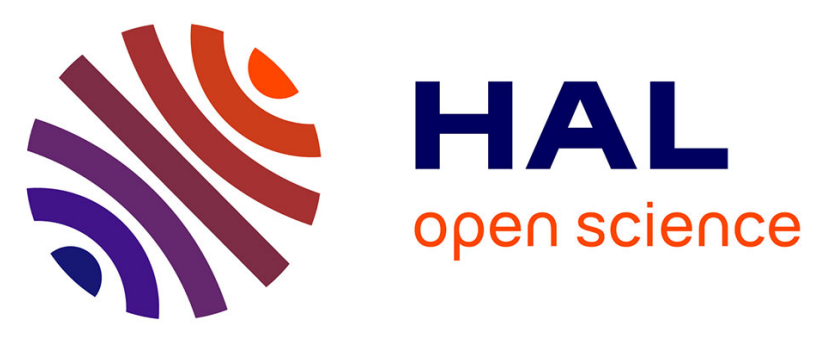

\title{
Robust dynamic analysis of detuned-mistuned rotating bladed disks with geometric nonlinearities
}

\author{
Anthony Picou, Evangéline Capiez-Lernout, Christian Soize, M. Mbaye
}

\section{To cite this version:}

Anthony Picou, Evangéline Capiez-Lernout, Christian Soize, M. Mbaye. Robust dynamic analysis of detuned-mistuned rotating bladed disks with geometric nonlinearities. Computational Mechanics, 2020, 65 (3), pp.711-730. 10.1007/s00466-019-01790-4 . hal-02460877

\section{HAL Id: hal-02460877 https://hal.science/hal-02460877}

Submitted on 30 Jan 2020

HAL is a multi-disciplinary open access archive for the deposit and dissemination of scientific research documents, whether they are published or not. The documents may come from teaching and research institutions in France or abroad, or from public or private research centers.
L'archive ouverte pluridisciplinaire HAL, est destinée au dépôt et à la diffusion de documents scientifiques de niveau recherche, publiés ou non, émanant des établissements d'enseignement et de recherche français ou étrangers, des laboratoires publics ou privés. 


\title{
Robust dynamic analysis of detuned-mistuned rotating bladed disks with geometric nonlinearities
}

\author{
A. Picou ${ }^{\mathrm{a}, \mathrm{b}}$, E. Capiez-Lernout ${ }^{\mathrm{a}}$, C. Soize ${ }^{*, a}$, M. Mbaye ${ }^{\mathrm{b}}$ \\ ${ }^{a}$ Université Paris-Est Marne-la-Vallée, MSME UMR 8208 CNRS, 5 bd Descartes, 77454 Marne-La-Vallée, France \\ ${ }^{b}$ Safran Tech, Modeling and Simulation, Rue des Jeunes Bois, Châteaufort, Magny-Les-Hameaux, 78772, France
}

\begin{abstract}
This work is devoted to the robust analysis of the effects of geometric nonlinearities on the nonlinear dynamic behavior of rotating detuned (intentionally mistuned) bladed disks in presence of unintentional mistuning (simply called mistuning). Mistuning induces uncertainties in the computational model, which are taken into account by a probabilistic approach. This paper presents a series of novel results of the dynamic behavior of such rotating bladed disks exhibiting nonlinear geometric effects. The structural responses in the time domain are analyzed in the frequency domain. The frequency analysis exhibits responses outside the frequency band of excitation. The confidence region of the stochastic responses allows the robustness to be analyzed with respect to uncertainties and also allows physical insights to be given concerning the structural sensitivity. The bladed disk structure is made up of 24 blades for which several different detuned patterns are investigated with and without mistuning.
\end{abstract}

Key words: Mistuning, Detuning, Bladed disks, Dynamics, Geometric nonlinearities, Uncertainty Quantification

\section{Introduction}

The intentional mistuning, also called detuning, has been identified as an efficient technological way for reducing the sensitivity of the forced response of bladed disks to unintentional mistuning (simply called mistuning), caused by the manufacturing tolerances and the small variations in the mechanical properties from blade to blade [1, 2, 3]. The objective of the detuning is to reduce the sensitivity of the response amplification induced by the mistuning. This random amplification is defined as the random ratio between the random highest dynamic response of a given detuned bladed disk in presence of mistuning and the deterministic highest response of the tuned bladed disk under the same excitation. It has been proposed to detune the bladed disk structure by using partial or alternating patterns of different sector types. A sector is constituted of a blade and of the corresponding part of the disk.

This technology has intensively been studied in the framework of the linear dynamic analysis (see for instance, [4, 5, 6, 7, 8, 9, 10, 11, 12, 13, 14]), for which the mistuning effects have been modeled using either parametric probabilistic approaches (see for instance, [15, 16, 17, 18, 19]),

${ }^{*}$ Corresponding author: C. Soize, christian.soize@u-pem.fr

Preprint of the published paper in Computational Machanics, 2020, doi:10.1007/s00466-019-01790-4. 
or using the nonparametric probabilistic approach ([20, 21, 22]), and for which the optimization of alternating patterns has been studied (see for instance, [23, 24, 25, 26]).

More recently, the technological improvements that include the use of more flexible and lighter materials can lead to large displacements so that the linearization of the dynamic equations can no longer be used. In this context, the geometric nonlinearities have to be taken into account and there is a growing interest for including geometric nonlinearities in the dynamic analyses of detuned bladed disks. It should be noted that the effects of nonlinearities on the dynamics have been considered by [27] for a simple generic cyclic structure exhibiting nonlinear stiffness connections (and not for nonlinear geometrical effects, and furthermore, without mistuning). The effects of geometrical nonlinearities using the Harmonic Balance Method have been analyzed by [28, 29] for tuned bladed disks (with neither mistuning nor detuning). The first work concerning the effects of nonlinear geometrical effects of mistuned bladed disk based on a probabilistic model without detuning can be found in [30].

It should be noted that, in the framework of linear dynamics, the introduction of intentional mistuning induces a modification of the eigenfrequencies of the blades and allows the aerodynamic coupling to be reduced [31, 32, 33]. This type of result should be analyzed in nonlinear geometrical dynamics. Nevertheless, the aerodynamic coupling is not considered in this paper.

This work is devoted to the robust analysis of the effects of geometric nonlinearities on the nonlinear dynamic behavior of rotating bladed disks that are detuned in presence of mistuning. An ensemble of novel results are presented based on analyses performed with a stochastic computational model of a bladed disk structure consisting of 24 blades.

The methodology used for obtaining these novel results and the organization of the paper are presented below. Section 2 begins defining the boundary value problem of the nonlinear geometric dynamics for the detuned bladed disk in rotation taking into account geometric stiffness, gyroscopic coupling, and centrifugal stiffness matrices (that are zero matrices when the rotation speed is zero). The corresponding computational model is constructed by using the finite element method. This computational model is referred as the nonlinear high-fidelity computational model (NL-HFM). The robust analysis that is proposed cannot be performed using the NL-HFM because the number of degrees-of-freedom is much too large for analyzing such parameterized stochastic nonlinear dynamical system. Consequently, it is necessary to introduce an appropriate parameterized stochastic nonlinear reduced-order model for carrying out the robust analysis.

The methodology used for the construction of this appropriate nonlinear reduced-order model (NL-ROM) is presented. For this purpose, a modal basis is computed by solving the generalized eigenvalue problem associated with the NL-HFM for which the rotation speed is zero (therefore, without nonlinear geometrical effects, without damping, and without mistuning). A first nonlinear reduced-order model (NL-ROMF) is constructed by projecting the NL-HFM on the subspace spanned by this modal basis.

Another vector basis is then calculated using the Proper-Orthogonal Decomposition (POD) method [34, 35] applied to the nonlinear solution of the NL-ROMF, which is solved in the time domain. Note that this time solution is computed taking into account geometric stiffness, gyroscopic coupling, and centrifugal stiffness matrix, for a given speed of rotation.

A projection basis for the NL-HFM is obtained by composing the modal basis with the vector basis constructed with the POD method. The final nonlinear reduced-order model (NL-ROM) is then obtained by double projection of the NL-HFM that is to say, by projecting the NL-HFM on the subspace spanned by the projection basis introduced above. At the end of this section, we present the construction of the nonlinear stochastic reduced-order model (NL-SROM) that is based on the nonparametric probabilistic approach of uncertainties [36] that is implemented 
in the NL-ROM. This NL-SROM describes the stochastic nonlinear dynamics of the detuned rotating bladed disk in presence of mistuning. The NL-SROM is solved in the time domain using the Monte-Carlo method. The random quantities of interest, related to the nonlinear stochastic responses, are analyzed in both time and frequency domains.

Section 3 is devoted to the construction of the NL-SROM for the robust analysis of the detuned bladed disk in rotation in presence of mistuning. Sections 4 to 6 deal with the robust analysis of the 24-blades disk, including the convergence aspects.

\section{Mean (or nominal) nonlinear reduced-order model of a detuned bladed disk in rotation without mistuning}

\subsection{Assumptions and terminology}

For the sake of clarity, one has to distinguish the following terms:

1. The tuned structure is related to the conceptual structure, which exhibits a perfect $M$-order cyclic symmetry. For this configuration as denoted as $\mathcal{P}_{0}$, the geometry, the constitutive equation of material, and the boundary conditions related to the reference sector are invariant under the $\frac{2 \pi}{M}$ rotation around its axis of symmetry. A dynamic analysis can then be performed by using only one reference sector with appropriate phase-lag conditions on the boundary. In this work, the tuned bladed disk is analyzed in 3D as for the detuned bladed disk.

2. The detuned (or intentionally mistuned) structure is related to the conceptual structure for which there is a spatial distribution of different types of sectors that are characterized by a given pattern. Here, we will only use two sector types with identical geometry and for which the material properties of the blades are different. The detuned structure is defined by an assembly of these two sector types. For $M=24$ blades, a pattern will be defined, for instance, by $12 B 6 A 3 B 3 A$, which consists of 12 consecutive blades of type $B, 6$ of type $A, 3$ of type $B$, and 3 of type $A$.

3. The mistuned structure is related to the real structure for which the cyclic symmetry is broken and is defined at the beginning of this Section. It is modeled by using the nonparametric probabilistic approach of uncertainties [36].

It is assumed that:

(1) The bladed disk is made up of a linear elastic material.

(2) In the time domain, the amplitude of the external forces are assumed to be sufficiently large so that the structure undergoes geometric nonlinear effects induced by large displacements and strains.

(3) The bladed disk is in rotation around its rotational axis at a constant rotation speed $\Omega(\mathrm{rad} / \mathrm{s})$.

\subsection{Nonlinear boundary value problem}

We are interested in considering the nonlinear boundary value problem of the detuned bladed disk in rotation. A total Lagrangian formulation is chosen and the nonlinear dynamic equations are expressed in the rotating frame with respect to the reference configuration. The rotation axis of the bladed disk is defined as $\left(O, \mathbf{e}_{3}\right)$. Let $\mathcal{R}$ be the rotating referential cartesian coordinates system and let $\left(O, \mathbf{e}_{1}, \mathbf{e}_{2}, \mathbf{e}_{3}\right)$ be its related basis. Let $\mathcal{D}$ be the three-dimensional bounded open domain 
corresponding to such reference configuration and subjected to the body force field $\mathbf{g}(\mathbf{x}, t)=$ $\left(g_{1}(\mathbf{x}, t), g_{2}(\mathbf{x}, t), g_{3}(\mathbf{x}, t)\right)$, in which $\mathbf{x}=\left(x_{1}, x_{2}, x_{3}\right)$ denotes the position of a given point belonging to domain $\mathcal{D}$. The boundary $\partial \mathcal{D}$ is such that $\partial \mathcal{D}=\Gamma \cup \Sigma$ with $\Gamma \cap \Sigma=\emptyset$. The external unit normal to boundary $\partial \mathcal{D}$ is denoted by $\mathbf{n}=\left(n_{1}, n_{2}, n_{3}\right)$. The boundary part $\Gamma$ corresponds to the fixed part of the structure (in the local rotating frame) whereas the boundary part $\Sigma$ is subjected to the external surface force field $\mathbf{G}(\mathbf{x}, t)=\left(G_{1}(\mathbf{x}, t), G_{2}(\mathbf{x}, t), G_{3}(\mathbf{x}, t)\right)$. Note that the external force fields are derived from the Lagrangian transport into the reference configuration of the physical body/surface force fields applied in the deformed configuration. We then introduce the $(3 \times 3)$

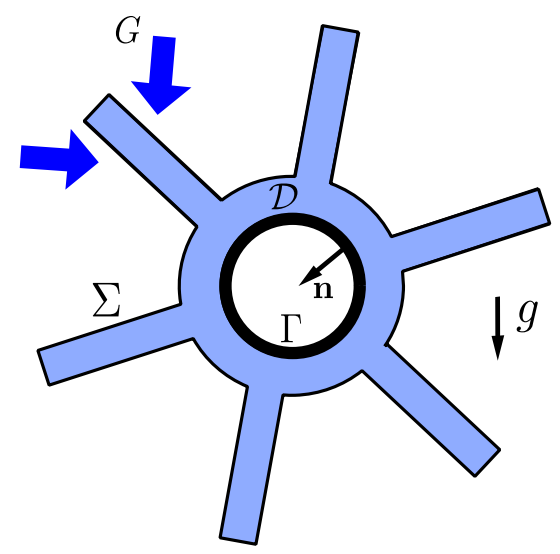

Figure 1: Scheme of the bladed disk in its reference configuration

rotation matrix $[R(\Omega)]$ such as

$$
[R(\Omega)]=\left[\begin{array}{ccc}
0 & -\Omega & 0 \\
\Omega & 0 & 0 \\
0 & 0 & 0
\end{array}\right]
$$

corresponding to the rotational axis $(0,0,1)$ in $\mathcal{R}$. From now on, the convention of summation over repeated latin indices is used. The unknown displacement field in $\mathcal{R}$ is denoted as $\mathbf{u}(\mathbf{x}, t)=$ $\left(u_{1}(\mathbf{x}, t), u_{2}(\mathbf{x}, t), u_{3}(\mathbf{x}, t)\right)$ and is solution of the following nonlinear boundary value problem [37]. For $i=1,2,3$, we have

$$
\begin{gathered}
\frac{\partial}{\partial x_{j}}\left(\mathbb{F}_{i k} \square_{k j}\right)+\rho g_{i}-\alpha \rho \frac{\partial u_{i}}{\partial t}=\rho \frac{\partial^{2} u_{i}}{\partial t^{2}}+2 \rho[R]_{i j} \frac{\partial u_{j}}{\partial t}+\rho[R]_{i j}[R]_{j k}\left(x_{k}+u_{k}\right), \forall \mathbf{x} \in \mathcal{D}, \\
\mathbb{F}_{i k} \square_{k j} n_{j}=G_{i}, \forall \mathbf{x} \in \Sigma, \\
u_{i}=0, \forall \mathbf{x} \in \Gamma,
\end{gathered}
$$


in which $\rho$ is the mass density and where $\alpha$ is the coefficient controlling the dissipation. In Eq. (2), the deformation gradient tensor $\mathbb{F}$ is defined by

$$
\mathbb{F}_{i j}=\frac{\partial u_{i}}{\partial x_{j}}+\delta_{i j},
$$

where $\delta_{i j}$ is the second-order unit tensor such that $\delta_{i j}=1$ if $i=j$ and 0 otherwise. The quantity $\square$ is the second Piola Kirchhoff symmetric stress tensor for which the constitutive equation for a linear elastic material is written as

$$
\mathbb{\square}_{i j}=\mathbb{a}_{i j k \ell} \mathbb{E}_{k \ell} .
$$

In Eq. (6), $₫$ is the fourth-order elasticity tensor and $\mathbb{E}$ is the Green strain tensor that is written as

$$
\mathbb{E}_{i j}=\frac{1}{2}\left(\frac{\partial u_{i}}{\partial x_{j}}+\frac{\partial u_{j}}{\partial x_{i}}+\frac{\partial u_{s}}{\partial x_{i}} \frac{\partial u_{s}}{\partial x_{j}}\right) .
$$

It should be noted that Eq. (6) captures the finite displacements of the structure. In the present context, it is assumed that there is no rigid body motion of the rotor and that the bladed disk structure rotates around a fixed axis. Such assumptions then allows for obtaining all the above equations as can be shown in [38, 37, 39, 40].

\subsection{Nonlinear high-fidelity model (NL-HFM) for a detuned bladed disk}

The finite element discretization of the weak formulation of the nonlinear boundary value problem defined by Eqs. (2) to (7) yields the following NL-HFM,

$$
[M] \ddot{\mathbf{u}}(t)+\left([D]+\left[C_{g}(\Omega)\right]\right) \dot{\mathbf{u}}(t)+[K(\Omega)] \mathbf{u}(t)+\mathbf{f}^{\mathrm{NL}}(\mathbf{u}(t))=\mathbf{f}(t),
$$

in which the $(n \times n)$ matrix $[K(\Omega)]$ is defined by

$$
[K(\Omega)]=\left[K_{e}\right]+\left[K_{c}(\Omega)\right]+\left[K_{g}(\Omega)\right],
$$

and is assumed to be positive definite. In Eq. [8], the $\mathbb{R}^{n}$-vector $\mathbf{u}(\mathrm{t})$ is the vector of the $n$ degreesof-freedom corresponding to the unknown displacements. The mass, damping, and stiffness $(n \times n)$ real matrices $[M],[D],\left[K_{e}\right]$ are positive definite, the geometric stiffness $(n \times n)$ real matrix $\left[K_{g}(\Omega)\right]$ is symmetric, the gyroscopic coupling $(n \times n)$ real matrix $\left[C_{g}(\Omega)\right]$ is skew-symmetric, and the centrifugal stiffness $(n \times n)$ real matrix $\left[K_{c}(\Omega)\right]$ is negative semi-definite. More precisely, matrix $\left[K_{g}(\Omega)\right]$ is associated with the term $\rho[R]_{i j}[R]_{j k} x_{k}$ in Eq. $[2]$, matrix $\left[K_{c}(\Omega)\right]$ with the term $\rho[R]_{i j}[R]_{j k} u_{k}$, and matrix $\left[C_{g}(\Omega)\right]$ with the term $2 \rho[R]_{i j} \frac{\partial u_{j}}{\partial t}$. The $\mathbb{R}^{n}$-vector $\mathbf{f}(t)$ is the external force vector depending on time issued from the finite element discretization of the surface and body force fields. The $\mathbb{R}^{n}$-vector $\mathbf{f}^{\mathrm{NL}}(\mathbf{u}(t))$ describes the nonlinear internal forces induced by the geometric nonlinearities.

The corresponding linear high-fidelity model (L-HFM) is defined similarly to Eq. (8) by removing the nonlinear term $\mathbf{f}^{\mathrm{NL}}(\mathbf{u}(t))$ and is written as

$$
[M] \ddot{\mathbf{u}}(t)+\left([D]+\left[C_{g}(\Omega)\right]\right) \dot{\mathbf{u}}(t)+[K(\Omega)] \mathbf{u}(t)=\mathbf{f}(t) .
$$




\subsection{Nonlinear reduced-order model (NL-ROM) for a detuned bladed disk}

The objective of this Section is to construct a nonlinear reduced-order model for a detuned bladed-disk. Such a construction requires the use of a vector basis for projecting the nonlinear computational model. Many methods can be used for constructing such vector basis, see for instance [41, 42, 30, 43, 29].

In this paper, the methodology proposed for constructing the NL-ROM is a novel approach, briefly described in Section 1 and that we detail hereinafter. The construction of the NL-ROM requires the knowledge of a consistent vector basis. It is first chosen of computing the vibrational modes of the linear high fidelity model (L-HFM) that includes the rotation terms but for which the damping term represented by matrix $[D]$ is removed and for which the gyroscopic coupling terms are not considered, mainly to avoid the computation of a complex eigenvalue problem for very large dynamical systems and to avoid the use of a complex basis for constructing the NL-ROM. This vector basis is not an optimal one with respect to the convergence speed of the reducedorder model since it ignores the nonlinear geometrical effects and the gyroscopic coupling terms (the damping matrix $[D]$ does not influence the convergence speed because damping is very small). This intermediate NL-ROMF is nevertheless constructed in order to carefully study the convergence of the nonlinear response related to the detuned rotating bladed-disk structure with all the rotating terms, the nonlinear geometrical effects, and the damping term. Such converged nonlinear solution that is computed in the time domain is then used to calculate another real vector basis using the Proper Orthogonal Decomposition method (POD-method). By combining these two vector bases, a second NL-ROM is obtained with a reasonable size. Through this NL-ROM, (1) the damping effect and all the rotating effects are taken into account, (2) for the detuned rotating structure, the NL-ROM is of lower order, which is more efficient than the NLROMF in terms of computational costs, (3) the probabilistic model describing the mistuning can be implemented through the nonlinear stochastic reduced-order model (NL-SROM). In addition, it allows for decreasing the computational costs for the Monte Carlo numerical simulation using a parallel computer.

\subsubsection{First nonlinear reduced-order model (NL-ROMF) for a detuned bladed disk}

The finite element model of the detuned bladed disk will exhibit a large number of degreesof-freedom (dofs), for instance, 1 million, yielding a large NL-HFM. Since the objective of this work is to perform a robust analysis of this NL-HFM demanding an extensive parametric study with respect to a subsequent number of distinct patterns, it is essential to consider a nonlinear reduced-order model (NL-ROM). The construction of the corresponding NL-ROM requires the knowledge of a projection basis. As explained in Section 1. we have to construct a projection basis that is performed in two steps. For the first step, consisting in calculating a modal basis, a possible strategy would consist in solving the generalized eigenvalue problem related to the linear, conservative, and homogeneous problem associated with the differential equation (8), yielding complex eigenvectors because of the gyroscopic coupling matrix. To avoid this difficulty, the modal basis is chosen to be real and is built as follows. The eigenfrequencies $\bar{v}_{\alpha}$ and the corresponding elastic modes $\bar{\varphi}_{\alpha}$ are obtained by solving the following generalized eigenvalue problem,

$$
[K(\Omega)] \bar{\varphi}_{\alpha}=\bar{\lambda}_{\alpha}[M] \bar{\varphi}_{\alpha}
$$

with $\alpha=\{1, \ldots, m\}$, where the eigenvalues $\bar{\lambda}_{\alpha}=\left(2 \pi \bar{v}_{\alpha}\right)^{2}$ are such that $0<\bar{\lambda}_{1} \leqslant \bar{\lambda}_{2} \leqslant \cdots \leqslant \bar{\lambda}_{m}$, and where the elastic modes $\bar{\varphi}_{1}, \ldots, \bar{\varphi}_{m}$ are stored in the $(n \times m)$ real modal matrix $\left[\bar{\Phi}^{m}\right]$ that is such that $\left[\bar{\Phi}^{m}\right]^{T}[M]\left[\bar{\Phi}^{m}\right]=\left[I_{m}\right]$. 
The first nonlinear reduced-order model (NL-ROMF), as proposed in Section 1 , is obtained by projecting Eq. (8) on the subspace generated by $\left[\bar{\Phi}^{m}\right]$ and is written as

$$
\begin{gathered}
\mathbf{u}(t)=\left[\bar{\Phi}^{m}\right] \overline{\mathbf{q}}(t), \\
{[\overline{\mathcal{M}}] \ddot{\ddot{\mathbf{q}}}(t)+\left([\overline{\mathcal{D}}]+\left[\overline{\mathcal{C}}_{g}(\Omega)\right]\right) \dot{\mathbf{q}}(t)+[\overline{\mathcal{K}}(\Omega)] \overline{\mathbf{q}}(t)+\overline{\mathcal{F}}^{\mathrm{NL}}(\overline{\mathbf{q}}(t))=\overline{\mathcal{F}}(t),}
\end{gathered}
$$

in which $\overline{\mathbf{q}}(t)$ is the $\mathbb{R}^{m}$-vector of the generalized coordinates and where $[\overline{\mathcal{M}}],[\overline{\mathcal{D}}],\left[\overline{\mathcal{C}}_{g}(\Omega)\right]$, and $[\overline{\mathcal{K}}(\Omega)]$ are the $(m \times m)$ reduced mass, damping, gyroscopic, and stiffness matrices, which are deduced from $[M],[D],\left[C_{g}(\Omega)\right]$, and $[K(\Omega)]$. In Eq. [13), the $\mathbb{R}^{m}$-vector $\overline{\mathcal{F}}(t)$ is the vector of the reduced external forces depending on time. The $\mathbb{R}^{m}$-vector $\overline{\mathcal{F}}^{\mathrm{NL}}(\overline{\mathbf{q}}(t))$ is the vector of the nonlinear reduced internal forces defined by

$$
\overline{\mathcal{F}}^{\mathrm{NL}}(\overline{\mathbf{q}}(t))=\left[\bar{\Phi}^{m}\right]^{T} \mathbf{F}^{\mathrm{NL}}\left(\left[\bar{\Phi}^{m}\right] \overline{\mathbf{q}}(t)\right) .
$$

\subsubsection{Linear reduced-order model (L-ROM) for a detuned bladed disk}

When the nonlinear reduced internal forces $\overline{\mathcal{F}}^{\mathrm{NL}}$ are removed from Eq. (13), the linear reduced-order model corresponding to Eqs. (12) and (13) is then denoted as L-ROM.

\subsubsection{Second nonlinear reduced-order model (NL-ROM) for a detuned bladed disk}

As explained in Section 11, the second step consists in using the POD-method applied to the NL-ROMF, which allows for taking into account the effects of the gyroscopic coupling.

Equation (13) is solved using a Newmark scheme for which a constant time step $\Delta t$ is used. At each time step, the nonlinear algebraic equation is solved using either the fixed point method or a continuation method based on the arc-length method depending on the local nonlinearity rate. Let $n_{t}$ be the number of time steps. It should be noted that the distance between two consecutive snapshots could be chosen as $r \Delta t$ where $r$ is a given integer greater than or equal to 1. Nevertheless, since the POD is applied to NL-ROMF, which has a small dimension $m \ll n$, it is not penalizing to take $r=1$. Let [A] be the $\left(m \times n_{t}\right)$ real matrix with $n_{t}>m$ defined by

$$
[A]_{i j}=\bar{q}_{i}\left(t_{j}\right) \sqrt{\Delta t},
$$

in which the constant time step $\Delta t$ is used as the distance between two consecutive snapshots for the construction of the POD basis. The vector basis is made up of the eigenvectors corresponding to largest eigenvalues of the $(m \times m)$ real matrix $[C]$ defined by

$$
[C]=[A][A]^{T} .
$$

It should be noted that the rank of matrix $[C]$ is $\bar{m}$ that is less than or equal to $m$ (if $\bar{m}<m$, then $[C]$ is not positive definite but only positive). In practice, matrix $[C]$ is not computed. Its eigenvalues and its eigenvectors are obtained by computing the singular value decomposition of matrix $[A]$ using an economy size algorithm [44]. Removing the zero singular values, this decomposition can be written as,

$$
[A]=[W][S][V]^{T},
$$


in which $[W]$ is the $(m \times \bar{m})$ real matrix with $\bar{m} \leq m$, where $[V]$ is the $\left(n_{t} \times \bar{m}\right)$ real matrix, and where the $(\bar{m} \times \bar{m})$ matrix $[S]$ contains all the $\bar{m}$ non-zeros singular values sorted by decreasing order $s_{1} \geqslant s_{2} \geqslant \cdots \geqslant s_{\bar{m}}>0$, which are the square-roots of the positive eigenvalues of matrix $[C]$. It can also be shown that the $\bar{m}$ columns of matrix $[W]$ are the corresponding eigenvectors of matrix $[C]$ associated with the positive eigenvalues and matrix $[W]$ is such that $[W]^{T}[W]=\left[I_{\bar{m}}\right]$. Let $[\mathcal{W}(m, N)]$ be the $(m \times N)$ matrix with $N \leqslant \bar{m}<m$ that contains the eigenvectors related to the $N$ greatest singular values $s_{\alpha}, \alpha=\{1, \cdots, N\}$, which is such that $[\mathcal{W}(m, N)]^{T}[\mathcal{W}(m, N)]=\left[I_{N}\right]$. Finally, the projection basis, represented by the $(m \times N)$ real matrix $\left[\Phi^{(m, N)}\right]$ that will be used for obtaining the NL-ROM, is constructed such that

$$
\left[\Phi^{(m, N)}\right]=\left[\bar{\Phi}^{m}\right]\left[\mathcal{W}^{(m, N)}\right],
$$

with $\left[\Phi^{(m, N)}\right]^{T}[M]\left[\Phi^{(m, N)}\right]=\left[I_{N}\right]$. The NL-ROM is then obtained by projecting the NL-HFM, that is to say, is written as

$$
\begin{gathered}
\mathbf{u}(t)=\left[\Phi^{(m, N)}\right] \mathbf{q}(t), \\
{[\mathcal{M}] \ddot{\mathbf{q}}(t)+\left([\mathcal{D}]+\left[\mathcal{C}_{g}(\Omega)\right]\right) \dot{\mathbf{q}}(t)+[\mathcal{K}(\Omega)] \mathbf{q}(t)+\mathcal{F}^{\mathrm{NL}}(\mathbf{q}(t))=\mathcal{F}(t),}
\end{gathered}
$$

in which $\mathbf{q}(t)$ is the $\mathbb{R}^{N}$-vector of the generalized coordinates, where $[\mathcal{K}(\Omega)]$ is the $(N \times N)$ matrix that is written as

$$
[\mathcal{K}(\Omega)]=\left[\mathcal{K}_{e}\right]+\left[\mathcal{K}_{c}(\Omega)\right]+\left[\mathcal{K}_{g}(\Omega)\right],
$$

and where $\left[\mathcal{K}_{e}\right],\left[\mathcal{K}_{c}(\Omega)\right]$, and $\left[\mathcal{K}_{g}(\Omega)\right]$ are the reduced elastic, centrifugal, and geometric matrices. In Eq. [20], the $(N \times N)$ real matrices $[\mathcal{M}],[\mathcal{D}],\left[\mathcal{C}_{g}(\Omega)\right]$, and $[\mathcal{K}(\Omega)]$ are the reduced matrices, which are deduced from $[M],[D],\left[C_{g}(\Omega)\right]$, and $[K(\Omega)]$. The normalization of matrix $\left[\Phi^{(m, N)}\right]$ is such that $[\mathcal{M}]=\left[I_{N}\right]$. The $\mathbb{R}^{N}$-vector $\mathcal{F}(t)$ is the generalized external forces. In Eq. [20], the $\mathbb{R}^{N}$-vector $\mathcal{F}^{\mathrm{NL}}(\mathbf{q}(t))$ of the nonlinear internal forces are written, for all $\mathbf{q}=\left(q_{1}, \ldots, q_{N}\right)$ in $\mathbb{R}^{N}$, as

$$
\mathcal{F}_{\alpha}^{\mathrm{NL}}(\mathbf{q})=\mathcal{K}_{\alpha \beta \gamma}^{(2)} q_{\beta} q_{\gamma}+\mathcal{K}_{\alpha \beta \gamma \delta}^{(3)} q_{\beta} q_{\gamma} q_{\delta},
$$

in which the quadratic and cubic stiffness contributions $\mathcal{K}_{\alpha \beta \gamma}^{(2)}$ and $\mathcal{K}_{\alpha \beta \gamma \delta}^{(3)}$ are written [45, 46] as

$$
\mathcal{K}_{\alpha \beta \gamma}^{(2)}=\frac{1}{2}\left(\widehat{\mathcal{K}}_{\alpha \beta \gamma}^{(2)}+\widehat{\mathcal{K}}_{\beta \gamma \alpha}^{(2)}+\widehat{\mathcal{K}}_{\gamma \alpha \beta}^{(2)}\right)
$$

with

$$
\begin{gathered}
\widehat{\mathcal{K}}_{\alpha \beta \gamma}^{(2)}=\int_{\mathbf{\Omega}} a_{j k \ell m} \varphi_{j, k}^{\alpha} \varphi_{s, \ell}^{\beta} \varphi_{s, m}^{\gamma} \mathrm{d} \mathbf{x}, \\
\mathcal{K}_{\alpha \beta \gamma \delta}^{(3)}=\frac{1}{2} \int_{\mathbf{\Omega}} a_{j k \ell m} \varphi_{r, j}^{\alpha} \varphi_{r, k}^{\beta} \varphi_{s, \ell}^{\gamma} \varphi_{s, m}^{\delta} \mathrm{d} \mathbf{x},
\end{gathered}
$$

in which $\varphi_{j}^{\alpha}$ corresponds to the entry $\left[\Phi^{(m, N)}\right]_{j \alpha}$. Note that tensor $\mathcal{K}_{\alpha \beta \gamma}^{(2)}$ has permutation-invariance property and that tensor $\mathcal{K}_{\alpha \beta \gamma \delta}^{(3)}$ has positive-definiteness property. The notation $y_{r, j}$ means the partial derivative of $y_{r}$ with respect to $x_{j}$. 


\section{Stochastic linear and nonlinear reduced-order models of a detuned rotating bladed disk with mistuning}

In this section, we introduce two probabilistic models for NL-ROM, yielding two nonlinear stochastic nonlinear reduced-order models, NL-SROM1 and NL-SROM2. The NL-SROM2 is introduced in order to compare it with L-SROM that will be constructed as the NL-SROM2 without the nonlinear internal forces, while NL-SROM1 will be the full probabilistic model for the nonlinear case.

\subsection{Stochastic nonlinear reduced-order model NL-SROM1 of a detuned rotating bladed disk with mistuning}

The first stochastic nonlinear reduced-order model (NL-SROM1) is based on a full probabilistic model and corresponds to a probabilistic modeling of the mistuning for the detuned rotating bladed disk. Note that only the nonlinear internal forces (including the linear elastic part) are assumed to be uncertain and consequently, are modeled by random quantities. As previously explained, the nonparametric probabilistic approach for geometric nonlinearities [45, 36] is used. It involves a positive $\left(N_{a} \times N_{a}\right)$ real matrix $\left[\mathcal{K}^{\mathrm{NL}}\right]$ with $N_{a}=N(N+1)$ which is written as

$$
\left[\mathcal{K}^{\mathrm{NL}}\right]=\left[\begin{array}{cc}
{\left[\mathcal{K}_{e}\right]} & {\left[\widetilde{\mathcal{K}}^{(2)}\right]} \\
{\left[\widetilde{\mathcal{K}}^{(2)}\right]^{T}} & 2\left[\widetilde{\mathcal{K}}^{(3)}\right]
\end{array}\right],
$$

in which $\left[\mathcal{K}_{e}\right]$ is the reduced elastic matrix defined in Eq. $(21)$ and where $\left[\widetilde{\mathcal{K}}^{(2)}\right]$ and $\left[\widetilde{\mathcal{K}}^{(3)}\right]$ are respectively the $\left(N \times N^{2}\right)$ and $\left(N^{2} \times N^{2}\right)$ real matrices resulting from the following reshaping operation,

$$
\left[\widetilde{\mathcal{K}}^{(2)}\right]_{\alpha J}=\widehat{\mathcal{K}}_{\alpha \gamma \delta}^{(2)} \quad, \quad\left[\widetilde{\mathcal{K}}^{(3)}\right]_{I J}=\mathcal{K}_{\alpha \beta \gamma \delta}^{(3)}
$$

with $I=(\alpha-1) N+\beta$ and $J=(\gamma-1) N+\delta$. The corresponding random matrix $\left[\mathcal{K}^{\mathrm{NL}}\right]$ is then written as

$$
\left[\mathcal{K}^{\mathrm{NL}}\right]=\left[L_{K}\right]\left[\mathbf{G}_{K}\left(\delta_{K}\right)\right]\left[L_{K}\right]^{T}+[\Delta \widetilde{\mathcal{K}}],
$$

in which $\left[L_{K}\right]$ is a $\left(N_{a} \times N_{G}\right)$ real matrix whose columns contain the $N_{G}$ eigenvectors of matrix $\left[\mathcal{K}^{\mathrm{NL}}\right]$ associated with the first $N_{G}$ largest eigenvalues. Such factorization allows for reducing the size of the random matrix $\left[\mathbf{G}_{K}\left(\delta_{K}\right)\right]$, as proposed in [47]. The full $\left(N_{G} \times N_{G}\right)$ random matrix $\left[\widetilde{\mathbf{G}}_{K}\left(\delta_{K}\right)\right]$ with $N_{G} \ll N_{a}$ is constructed using the Maximum Entropy principle [48, 36]. In Eq. [28), the $\left(N_{a} \times N_{a}\right)$ real matrix $[\Delta \widetilde{\mathcal{K}}]$ is written as

$$
[\Delta \widetilde{\mathcal{K}}]=\left[\mathcal{K}^{\mathrm{NL}}\right]-\left[L_{K}\right]\left[L_{K}\right]^{T},
$$

and $E\left\{\left[\mathcal{K}^{\mathrm{NL}}\right]\right\}=\left[\mathcal{K}^{\mathrm{NL}}\right]$ because $E\left\{\left[\widetilde{\mathbf{G}}_{K}\left(\delta_{K}\right)\right]\right\}=\left[I_{N_{G}}\right]$. The hyperparameter $\delta_{K}$ allows for controlling the level of uncertainties in random matrix $\left[\mathcal{K}^{\mathrm{NL}}\right]$. The random linear, quadratic, and cubic coefficients, $\left[\mathcal{K}_{e}\right]_{\alpha \beta}, \mathcal{K}_{\alpha \beta \gamma}^{(2)}$, and $\mathcal{K}_{\alpha \beta \gamma \delta}^{(3)}$, are extracted from random matrix $\left[\mathcal{K}^{\mathrm{NL}}\right]$ that have the same block structure as its deterministic counterpart defined in Eq. (26). The first stochastic nonlinear reduced-order model, NL-SROM1, is then written as,

$$
\mathbf{U}(t)=\left[\Phi^{(m, N)}\right] \mathbf{Q}(t),
$$




$$
\begin{aligned}
{[\mathcal{M}] \ddot{\mathbf{Q}}(t)+\left([\mathcal{D}]+\left[\mathcal{C}_{g}(\Omega)\right]\right) \dot{\mathbf{Q}}(t)+\left(\left[\mathcal{K}_{e}\right]+\left[\mathcal{K}_{c}(\Omega)\right]+\left[\mathcal{K}_{g}(\Omega)\right]\right) } & \mathbf{Q}(t) \\
& +\mathcal{F}^{\mathrm{NLS}}(\mathbf{Q}(t))=\mathcal{F}(t),
\end{aligned}
$$

in which $\mathbf{Q}(t)$ is the $\mathbb{R}^{N}$-valued random variable. In Eq. (31), the vector of the stochastic nonlinear internal forces $\mathcal{F}^{\mathrm{NLS}}(\mathbf{Q}(t))$ is written, for all $\mathbf{q}=\left(q_{1}, \ldots, q_{N}\right)$ in $\mathbb{R}^{N}$, as

$$
\mathcal{F}_{\alpha}^{\mathrm{NLS}}(\mathbf{q})=\mathcal{K}_{\alpha \beta \gamma}^{(2)} q_{\beta} q_{\gamma}+\mathcal{K}_{\alpha \beta \gamma \delta}^{(3)} q_{\beta} q_{\gamma} q_{\delta} .
$$

3.2. Stochastic nonlinear reduced-order model NL-SROM2 of a detuned rotating bladed disk with mistuning

The second nonlinear stochastic reduced-order model, NL-SROM2, is defined by Eqs. (30) and (31) for which two modifications are performed. Firstly, the stochastic nonlinearity term $\mathcal{F}^{\mathrm{NLS}}$ in Eq. (31) is replaced by the deterministic nonlinearity term $\mathcal{F}^{\mathrm{NL}}$ defined by Eq. (22). Secondly, another probabilistic model is introduced for the random positive-definite $(N \times N)$ matrix $\left[\mathcal{K}_{e}\right]$, which is written as

$$
\left[\mathcal{K}_{e}\right]=\left[L_{\mathcal{K}_{e}}\right]\left[\mathbf{G}_{K}\left(\delta_{K}\right)\right]\left[L_{\mathcal{K}_{e}}\right]^{T},
$$

in which $\left[L_{\mathcal{K}_{e}}\right]$ is the lower triangular $(N \times N)$ real matrix, which results from the Cholesky factorization of $(N \times N)$ real matrix $\left[\mathcal{K}_{e}\right]$, and where $\left[\mathbf{G}_{K}\left(\delta_{K}\right)\right]$ is the random $(N \times N)$ positivedefinite real matrix that is similarly constructed as $\left[\widetilde{\mathbf{G}}_{K}\left(\delta_{K}\right)\right]$.

\subsection{Stochastic linear reduced-order model L-SROM of a detuned rotating bladed disk with mis- tuning}

We also introduce a stochastic linear reduced-order model L-SROM, which is the NL-SROM2, in which the nonlinear term $\mathcal{F}^{\mathrm{NLS}}$ is removed.

\subsection{Observations of the nonlinear dynamical system}

Observations of the nonlinear dynamical system have to be defined for performing the robust analysis of the detuned rotating bladed disk with or without mistuning. It is recalled that, in presence of mistuning, the responses are random. There are several ways for defining the observations. We have chosen one, which is coherent with all the analyses that are performed in the frequency domain. First, we will define only one observation point for each blade, which is located at its tip. This means that, the number of observation points is equal to the number of blades. For the detuned rotating bladed disk without mistuning, we will look for the blade number $j_{0}$ where the maximum related to the amplitude of the displacement occurs, over all the blades and for the entire frequency band of analysis. It should be noted that, in presence of mistuning, $j_{0}$ becomes a random variable. Nevertheless, we want to characterize the random responses - of the detuned rotating bladed disk in presence of mistuning - with respect to the deterministic response of the detuned rotating bladed disk without mistuning. Consequently, we have chosen to keep $j_{0}$ as the deterministic blade number for the case for which mistuning is taken into account.

Figure 2 shows the computational model of the bladed disk for which, as previously explained, the $M$ observation points are located at the tip of each blade (red dots). We first consider the detuned rotating bladed disk without mistuning. For each time $t$ and for the observation in blade $j$, let $\mathbf{u}^{j}(t)=\left(u_{1}^{j}(t), u_{2}^{j}(t), u_{3}^{j}(t)\right)$ be the vector whose coordinates are given in the local basis 
$\left(\mathbf{e}_{1}^{j}, \mathbf{e}_{2}^{j}, \mathbf{e}_{3}^{j}\right)$. For frequency $v$ in Hz, the Fourier Transform of function $t \mapsto \mathbf{u}^{j}(t)$ is written as $\widehat{\mathbf{u}}^{j}(2 \pi v)=\left(\widehat{u}_{1}^{j}(2 \pi v), \widehat{u}_{2}^{j}(2 \pi v), \widehat{u}_{3}^{j}(2 \pi v)\right)$. We have to find the blade number $j_{0}$ such that

$$
j_{0}=\arg \max _{j=1, \ldots, M}\left\|\widehat{\mathbf{u}}^{j}\right\|
$$

in which $\left\|\left.\right|^{j} \mid\right\|$ is such that

$$
\left\|\widehat{\mathbf{u}}^{j}\right\|\left\|=\max _{v}\right\| \widetilde{\mathbf{u}}^{j}(2 \pi v) \|,
$$

with $\left\|\widehat{\mathbf{u}}^{j}(2 \pi v)\right\|^{2}=\left.\sum_{k=1}^{3} \widehat{u}_{k}^{j}(2 \pi v)\right|^{2}$. For the tuned rotating bladed disk (therefore, it is not detuned and there is no mistuning), the quantity $\left\|\widehat{\mathbf{u}}^{j}\right\| \mid$ will be rewritten as $\left\|\left.\right|_{\mathbf{u}} ^{\text {tuned }}\right\|$. It should be noted that, the blade number $j_{0}$ depends on the considered pattern and on the type of the analysis, which is performed for the detuned rotating bladed disk. There are two types of analysis, the linear one denoted by subscript $\mathrm{L}$ and the nonlinear one denoted by subscript NL. These subscripts will be omitted when no confusion will be possible.

We are interested in characterizing the amplification levels for the nonlinear deterministic and random cases. The random observations corresponding to the detuned-mistuned cases are similarly denoted by replacing lowercase letters by uppercase ones. We then define quantities $b(2 \pi v)$ and $B(2 \pi v)$ as the deterministic and random dynamic amplification factor such that

$$
\begin{aligned}
& b(2 \pi v)=\frac{\left\|\widehat{\mathbf{u}}^{j_{0}}(2 \pi v)\right\|}{\left\|\widehat{\mathbf{u}}^{\text {tuned }}\right\|}, \\
& B(2 \pi v)=\frac{\left\|\widehat{\mathbf{U}}^{j_{0}}(2 \pi v)\right\|}{\left\|\left.\right|_{\mathbf{u}} ^{\text {tuned }}\right\|},
\end{aligned}
$$

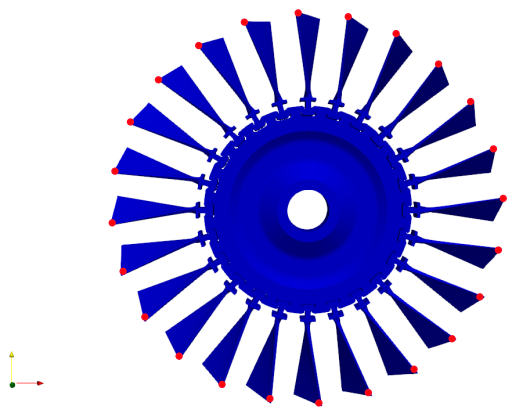

Figure 2: Finite element model of the bladed disk with 24 blades in which the dot symbols (red color) correspond to the excitation points (left figure). Zoom of the finite element model of a sector (right figure).

\subsection{Remark concerning the software implementation}

It should be noted that a commercial software is used for developing the finite element model of a given sector of the bladed-disk. All the other steps (constructing the computational model, 
computing the vector bases, constructing the LROM, the NL-ROM, the L-SROM and the NLSROM, computing the linear and nonlinear deterministic and stochastic dynamical responses) are processed using a house code. Since a house code is used, there is no problem for a direct implementation of the NL-ROM and NL-SROM (see [45, 46]).

\section{Robust analysis of the detuned bladed disk in rotation in presence of mistuning using the NL-SROM}

From an industrial point of view, such a robust analysis is of particular interest when exceptional operating ranges are considered (severe loads or close to a flutter situation). In such cases, the geometric nonlinearities can no longer be neglected and strongly modify the dynamical responses of the structure with respect to the usual linear case. The robust analysis presented will then correspond to such exceptional operating ranges.

\subsection{Finite element model of the tuned bladed disk with 24 blades}

The finite element model of the tuned bladed disk is shown in Figure 2. There are $M=24$ blades and the bladed disk rotates around its cyclic axis with a constant rotation speed $\Omega=$ $2 \pi \times 74 \mathrm{rad} / \mathrm{s}(4440 \mathrm{RPM})$. The material is steel, which is considered as a homogeneous and isotropic elastic material with Young modulus $2 \times 10^{11} \mathrm{~N} \times \mathrm{m}^{-2}$, Poisson's ratio 0.3 , and mass density $7650 \mathrm{Kg} \times \mathrm{m}^{-3}$. In the rotating frame, the disk is clamped at the inner radius of the disk (see Figure 11. The main geometric characteristics are summarized in Table 1 . The finite

\begin{tabular}{|c|c|}
\hline Inner disk radius & $19.8 \times 10^{-3} \mathrm{~m}$ \\
\hline Outer disk radius & $100 \times 10^{-3} \mathrm{~m}$ \\
\hline Disk width & $20 \times 10^{-3} \mathrm{~m}$ \\
\hline Blade thickness at root section & $4.8 \times 10^{-3} \mathrm{~m}$ \\
\hline Blade thickness at tip section & $2 \times 10^{-3} \mathrm{~m}$ \\
\hline
\end{tabular}

Table 1: Geometric characteristics of the bladed disk

element model of the reference sector has been carried out using tridimensional isoparametric solid finite elements with quadratic shape functions. The finite element mesh of a reference sector is constituted of 37488 hexahedral finite elements with 20 nodes, 1848 pyramidal elements with 13 nodes, and 45864 tetrahedral elements with 10 nodes. The finite element model of the full bladed disk is then obtained from the finite element model of the reference sector. The numerical description of the finite element model is given in Table 2. It should be noted that, for the linear

\begin{tabular}{|c|c|c|c|}
\hline Structure & Elements & Nodes & DOFs \\
\hline Blade alone & 2714 & 6896 & 20688 \\
\hline Disk sector & 836 & 4554 & 13662 \\
\hline Full structure & 85200 & 265080 & 787176 \\
\hline
\end{tabular}

Table 2: Element, nodes, and dofs of the finite element model

tuned rotating bladed disk, the eigenfrequency of the elastic mode that corresponds to the first flexural mode of the blade with a 4-nodal diameter is $v_{1}=435 \mathrm{~Hz}$. Following the damping model introduced in Eq. 22, the Rayleigh damping model is chosen in order that the critical damping rate be equal to $10^{-2}$ for the fundamental eigenfrequency $v_{1}$. 


\subsection{Definition of the patterns for the detuned bladed disk}

The computational model of the detuned bladed disk is constructed from the knowledge of two compatible meshes of two different sector types denoted as $A$ and $B$. The reference sector $B$ is obtained from sector $A$ by decreasing the Young modulus of the blade by $10 \%$; the Young modulus of the disk remains unchanged. Figure 3 shows the tuned system $\mathcal{P}_{0}=24 A$ and the detuned one $\mathcal{P}_{31}=(6 A 6 B)_{2}$.
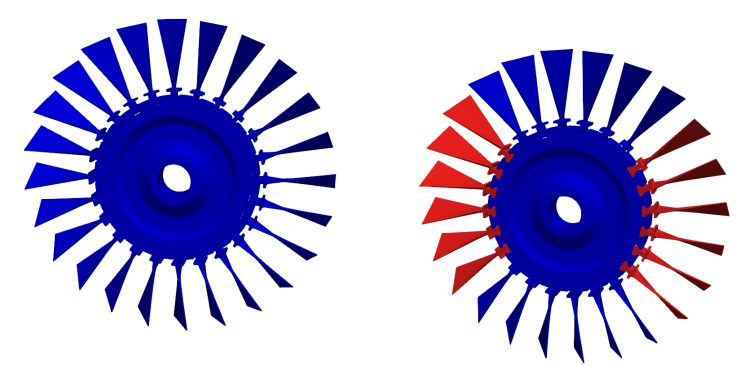

Figure 3: Tuned system $\mathcal{P}_{0}=24 A$ (left figure) and detuned system $\mathcal{P}_{31}=(6 A 6 B)_{2}$ with red blade for $B$ and blue blade for $A$ (right figure). For black and white printing, red color is light grey and blue color is black.

\subsection{Eigenfrequencies of the linear tuned rotating bladed disk}

Figure 4 displays the Campbell diagram representing the evolution of the eigenfrequencies $v_{\alpha}$ of the linear tuned rotating bladed disk $\mathcal{P}_{0}$ according to rotation speed $\Omega$. The dashed lines represent the $E O$-engine order excitation characterized by function $\Omega \mapsto E O \times \Omega / 60$. A required condition for an Engine Order (EO) excitation to excite a bladed-disk is that the EO frequency coincides with the natural frequency of the bladed-disk. It is then possible to graphically detect the possible resonant points. The intersection of the natural eigenfrequencies with the dashed lines gives then an indication of the rotating speed yielding resonant situations of interest. The EO excitation is a periodic force that can be given by

$$
j M \pm h=k \times E O,
$$

in which $j$ and $k$ are integers, and where $h$ is the circumferential wave number corresponding to the number of nodal diameters of the considered mode.

Figure 5 displays the graph of $v_{\alpha}(h)$ as a function of the circumferential wave number $h$ for the linear tuned rotating bladed disk (configuration $\mathcal{P}_{0}$ ) for which rotation speed is $4440 R P M$. For this rotation speed, we are interested in the first 3 modes related to $h=4$, which are the first bending mode of blades (mode 1 at $484 \mathrm{~Hz}$ ), the second bending mode of blades (mode 2 at $1170 \mathrm{~Hz}$ ), and the first torsion mode of blades (mode 3 at $1490 \mathrm{~Hz}$ ).

\subsection{Defining the external forces (excitation)}

The objective of the presented analysis is not to compute the nonlinear dynamical response for a general physical excitation, but is to present a sensitivity study for understanding the role played by the geometrical nonlinear effects with respect to the linear counterpart. In this framework, it should be noted that this choice is coherent with the fact that no aerodynamic coupling is 


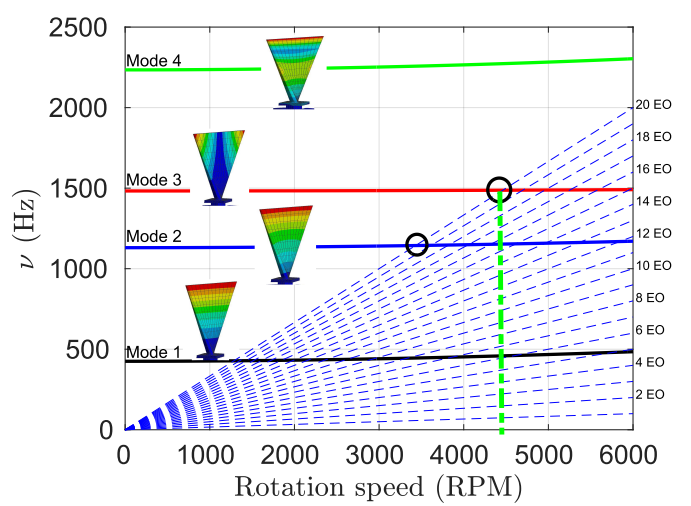

Figure 4: Graph of $\Omega \mapsto v_{\alpha}(\Omega)$ defining the Campbell diagram of the eigenfrequencies (in $\mathrm{Hz}$ ) of the linear tuned rotating bladed disk (pattern $\mathcal{P}_{0}$ ) as a function of the rotation speed (in RPM), where EO denotes the engine order, and where the vertical dashed line identifies the speed of rotation that is considered.

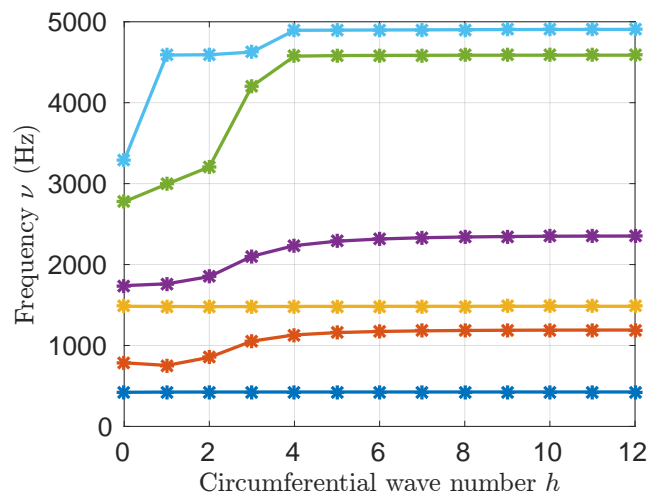

Figure 5: Graph of $h \mapsto v_{\alpha}(h)$ of the eigenfrequencies $v_{\alpha}$ of the linear tuned rotating bladed disk (pattern $\mathcal{P}_{0}$ ) for rotation speed $\Omega=4440 R P M$ as a function of the circumferential wave number $h$.

taken into account. Inspired by the type of analyses performed for the linear mistuned cases, the external forces have been chosen in order to control the circumferential wave number and also the frequency band of excitation, which has to be sufficiently narrow around the specified frequency of interest. For the role of the nonlinear effects, this type of excitation allows for clearly analyzing the transfer of energy outside the excitation frequency band (which is the objective of the paper).

According to the Campbell diagram displayed in Figure 4 it can be seen that the third mode intersect the EO line corresponding to $E O=20$ for the considered rotating speed $\Omega=4440 R P M$ (rotation per minute). As a consequence, the excitation is chosen with circumferential wave 
number $h=4$ (nodal diameter), for which Eq. (38) is satisfied with $j=k=1$.

The excitation frequency band is chosen as $\mathbb{B}_{e}=[1000,1600] \mathrm{Hz}$, which contains the first two eigenfrequencies corresponding to the flexural mode and the torsion mode of the blade as shown in Figure 4 . In the time domain, the external force vector $\mathbf{f}(t)$ is defined by,

$$
\mathbf{f}(t)=s_{0} g(t) \mathbf{f}^{s},
$$

in which $\mathbf{f}^{s}$ is the vector representing the spatial distribution of the external forces in $(\mathrm{N})$, which depends on the circumferential wave number $h$, and where the dimensionless time-function $t \mapsto$ $g(t)$ is defined on $\mathbb{R}$ and is constructed so that the modulus $|\widehat{g}(2 \pi v)|$ of its Fourier transform $\widehat{g}(2 \pi v)$ is equal to 1 in excitation frequency band $\mathbb{B}_{e}$ and equal to zero outside $\mathbb{B}_{e}$. The reference intensity of the force applied is represented by $g(t) \mathbf{f}^{s}(N)$ and the level of nonlinear geometric effects is driven by the dimensionless parameter $s_{0}$. For $s_{0}=0.01$, the nonlinear geometrical effects will be negligible, for $s_{0}=0.15$, the nonlinear geometrical effects will be moderate, and for $s_{0}=1$ the effects will be large. Note that the value $s_{0}=4$ has also been used for the sensitivity analysis and correspond to strong effects. Figures 6 and 7 show the graphs of function $t \mapsto g(t)$ and the modulus of its Fourier transform $v \mapsto|\widehat{g}(2 \pi v)|$. The frequency band of analysis is $\mathbb{B}_{a}=[0,4000] \mathrm{Hz}$. It should be noted that, from a computational point of view, the numerical values of $|\widehat{g}(2 \pi v)|$ for $v$ in $\mathbb{B}_{a} \backslash \mathbb{B}_{e}$ are not exactly zero but differ with three orders of magnitude lower, which means that the linear dynamic response will have negligible magnitude in the band $\mathbb{B}_{a} \backslash \mathbb{B}_{e}$ (that will be not the case for the nonlinear dynamic response). From a numerical point of view, function $g$ is truncated by choosing $t_{i n i}=-0.065 s$ such that $g\left(t_{i n i}\right)=0$ with a time duration $T=0.15 \mathrm{~s}$. The computations are carried out with $n_{t}=4096$ time steps, using a sampling frequency $v_{e}=16000 \mathrm{~Hz}$. The nonlinear dynamic analysis is performed in the time domain according to Eq. 201. A Fourier transform of the time response (a deterministic time response or a realization of the stochastic time response) is carried out and allows for analyzing a posteriori the nonlinear dynamic responses in the frequency domain (in a deterministic framework or in a stochastic one).

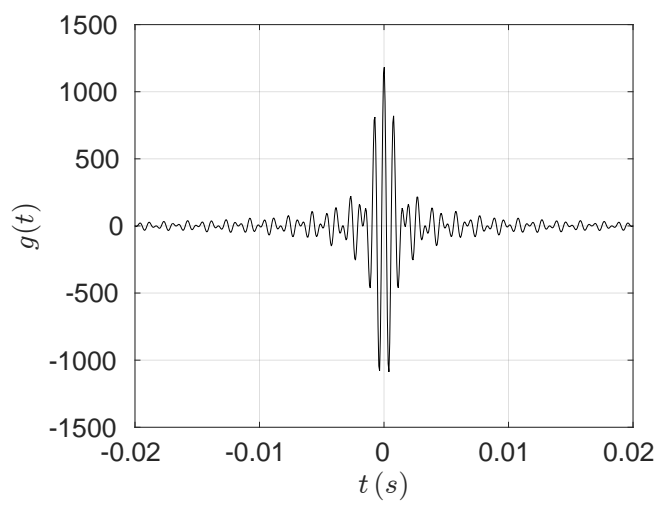

Figure 6: Zoom on the interval $[-0.02,0.02] s$ of the graph of the time-function excitation, $t \mapsto g(t)$, defines on interval $[-0.065,0.15] s$. 


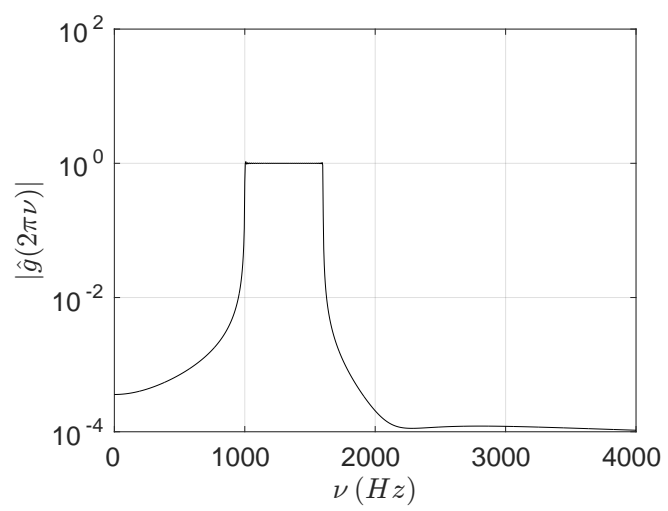

Figure 7: Graph of function $v \mapsto|\widehat{g}(2 \pi v)|$ in $\log$ scale.

4.5. Convergence analyses with respect to the parameters that control the reductions and the Monte-Carlo numerical simulations

\subsubsection{Convergence analysis with respect to the nonlinear reduced-order model}

In this section, the convergence analysis of the deterministic response of the NL-ROM is considered for a given pattern of a detuned rotating bladed disk (without mistuning). We estimate the optimal values of parameters $m$ and $N$ related to the truncation of the vector bases used for constructing the NL-ROM (according to Section 2.4.3). Let $\widehat{w}(2 \pi v)$ be the scalar value such that

$$
\widehat{w}(2 \pi v)=\sqrt{\sum_{j=1}^{M}\left\|\widehat{\mathbf{u}}^{j}(2 \pi v)\right\|^{2}} .
$$

When dealing with the first reduction, involving modal matrix $\left[\bar{\Phi}^{m}\right], \widehat{w}(2 \pi v)$ is denoted by $\widehat{w}^{m}(2 \pi v)$. When dealing with the final reduction, involving the modal matrix $\left[\Phi^{m, N}\right], \widehat{w}(2 \pi v)$ is denoted by $\widehat{w}^{m, N}(2 \pi v)$. A first convergence analysis is performed with respect to the number $m$ of modes to be kept in the NL-ROMF. Let $\operatorname{Conv}_{1}(m)$ be the function defined by

$$
\operatorname{Conv}_{1}(m)=\sqrt{\int_{\mathbb{B}_{a}}\left(\widehat{w}^{m}(2 \pi v)\right)^{2} d v} .
$$

Figure 8 displays the graph of function $m \mapsto \operatorname{Conv}_{1}(m)$ for the three different patterns (the tuned pattern $\mathcal{P}_{0}=24 A$ and two detuned patterns $\mathcal{P}_{2}=(A B)_{12}$ and $\mathcal{P}_{13}=6 B 12 A 3 B 3 A$ ). A good convergence is obtained for $m=145$ that will be the retained value. A second convergence analysis is then carried out with respect to $N<m=145$ according to Eq. [18). Let $\operatorname{Conv}_{2}(m, N)$ be the function defined by

$$
\operatorname{Conv}_{2}(m, N)=\sqrt{\int_{\mathbb{B}_{a}}\left(\widehat{w}^{m, N}(2 \pi v)\right)^{2} d v} .
$$




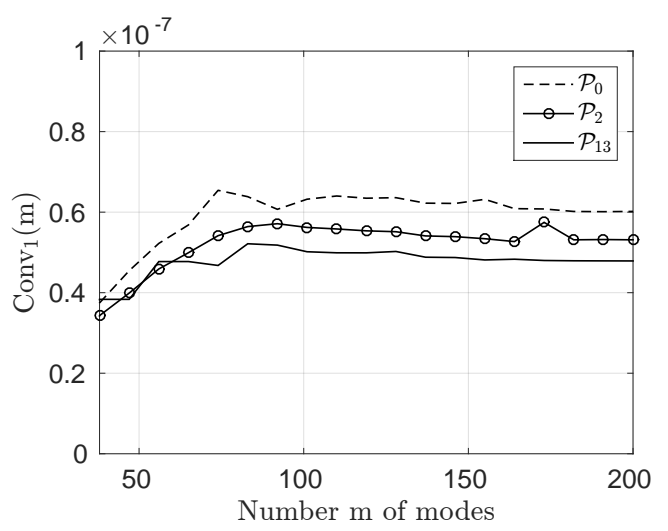

Figure 8: Convergence analysis with respect to the order $m$ of the NL-ROMF: graphs of function $m \mapsto \operatorname{Conv}_{1}(m)$ for patterns $\mathcal{P}_{0}=24 A, \mathcal{P}_{2}=(A B)_{12}$, and $\mathcal{P}_{13}=6 B 12 A 3 B 3 A$.

Figure 9 displays the graph of function $N \mapsto \operatorname{Conv}_{2}(m=145, N)$. It can be seen that a good approximation is obtained for $N=55$, which proves the efficiency of the reduction strategy that is proposed.

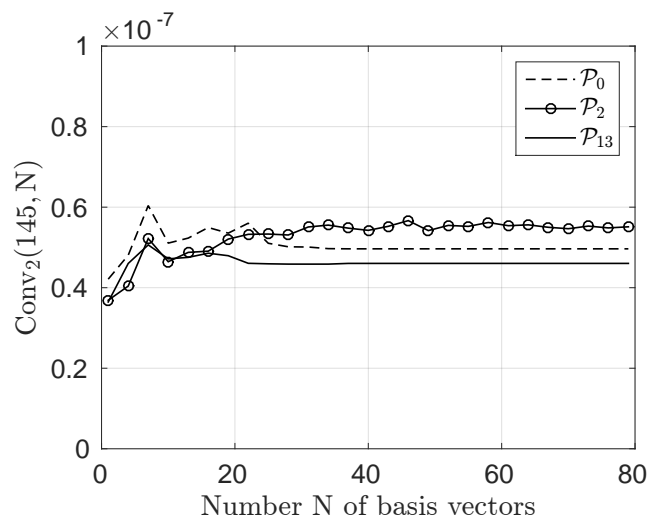

Figure 9: Convergence analysis with respect to the order $N$ of the NL-ROM for $m=145$ : graphs of function $N \mapsto \operatorname{Conv}_{2}(m, N)$ for patterns $\mathcal{P}_{0}=24 A, \mathcal{P}_{2}=(A B)_{12}$, and $\mathcal{P}_{13}=6 B 12 A 3 B 3 A$.

\subsubsection{Convergence analysis with respect to the number $N_{G}$ of columns of matrix $\left[L_{K}\right]$}

From the previous section, the order of the NL-ROM is $N=55$. This means that the dimension of matrix $\left[\mathcal{K}^{\mathrm{NL}}\right]$ defined in Eq. [26] is $N_{a}=3080$. Random matrix [ $\left.\mathcal{K}^{\mathrm{NL}}\right]$ is represented by Eqs. (28) and [29), which depends on the number $N_{G}$ of columns of matrix $\left[L_{K}\right]$. We then 
introduce the relative error function $N_{G} \mapsto \operatorname{err}\left(N_{G}\right)$ such that

$$
\operatorname{err}\left(\mathrm{N}_{\mathrm{G}}\right)=\sqrt{\frac{\|[\Delta \widetilde{\mathcal{K}}]\|_{F}^{2}}{\left\|\left[\mathcal{K}^{\mathrm{NL}}\right]\right\|_{F}^{2}},}
$$

in which $\|\cdot\|_{F}$ is the Frobenius norm. Figure 10 displays the graph of $N_{G} \mapsto \operatorname{err}\left(N_{G}\right)$. A good convergence is obtained for $N_{G}=500$, which allows for reducing the size of the random matrix $\left[\mathbf{G}_{K}\left(\delta_{K}\right)\right]$ used in Eq. (28).

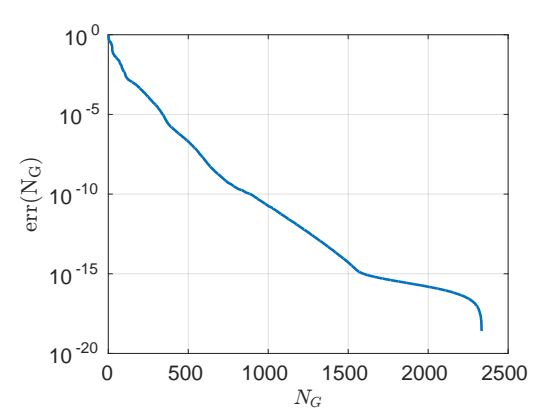

Figure 10: Convergence analysis with respect to the number of columns $N_{G}$ of matrix $\left[L_{K}\right]$ : graph of function $N_{G} \mapsto \operatorname{err}\left(N_{G}\right)$ in log-scale.

4.5.3. Convergence analysis of the NL-SROM with respect to the number of Monte-Carlo simulations

In this section, parameters $m, N$, and $N_{G}$ are such that $m=145, N=55$, and $N_{G}=500$. The dispersion parameter $\delta_{K}$ is fixed to the value 0.1 . Let

$$
\widehat{\mathcal{W}}(2 \pi v)=\sqrt{\sum_{j=1}^{M}\left\|\widehat{\mathbf{U}}^{j}(2 \pi v)\right\|^{2}}
$$

be the random variable corresponding to Eq. $(40)$ for the stochastic case. The stochastic equation (31) is solved by using the Monte-Carlo numerical simulation with $n_{s}$ realizations denoted by $\theta_{1}, \cdots, \theta_{n_{s}}$. Let $\widehat{\mathcal{W}}\left(2 \pi v, \theta_{\ell}\right)$ be the realization $\theta_{\ell}$ of the random variable $\widehat{\mathcal{W}}(2 \pi v)$. The convergence analysis with respect to $n_{s}$ is then carried out studying the function $n_{s} \mapsto \operatorname{Conv}_{4}\left(n_{s}\right)$ defined by

$$
\operatorname{Conv}_{4}\left(n_{s}\right)=\sqrt{\frac{1}{n_{s}} \sum_{\ell=1}^{n_{s}} \int_{\mathbb{B}_{a}}\left(\widehat{\mathcal{W}}\left(2 \pi v, \theta_{\ell}\right)\right)^{2} d v}
$$

In order to limit the CPU-time for performing the robust analysis of the detuned systems in presence of mistuning, we choose the value of $n_{s}$ to be 500, which corresponds to a reasonable compromise with respect to the level of convergence. 


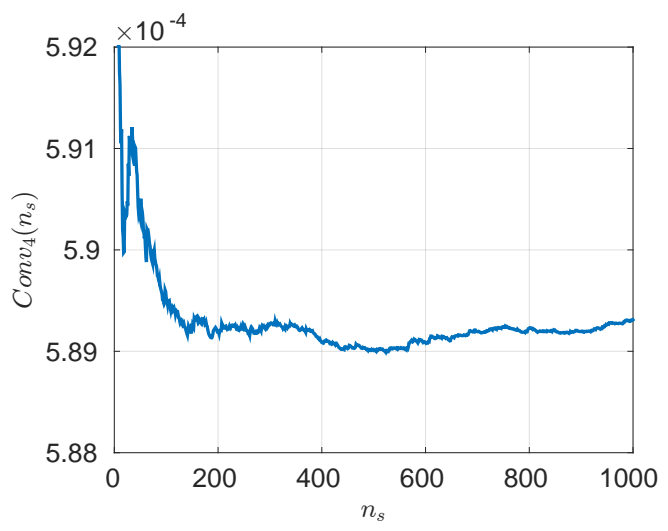

Figure 11: Convergence analysis with respect to the number $n_{s}$ of realizations for the MonteCarlo numerical simulation of the NL-SROM: graph of function $n_{s} \mapsto \operatorname{Conv}_{4}\left(n_{s}\right)$.

\section{Nonlinear deterministic analysis of nonlinear tuned and detuned rotating bladed disks without mistuning}

In all this section, we consider the nonlinear tuned rotating bladed disk and the nonlinear detuned one without mistuning, which are analyzed using the NL-ROMF and the NL-ROM.

\subsection{Sensitivity analysis of the deterministic responses for the nonlinear tuned rotating bladed disk with respect to $s_{0}$}

This external-forces sensitivity analysis is performed using the NL-ROMF with $m=145$ for the nonlinear tuned rotating bladed disk (pattern $\mathcal{P}_{0}=24 A$ ). The objective is to determine the value of parameter $s_{0}$ for which the geometric nonlinear effects occur in the dynamic response. This analysis is performed by quantifying the energy $i_{\mathrm{NL}}$ outside the excitation frequency band $\mathbb{B}_{e}$ (that is to say, in the band $\mathbb{B}_{a} \backslash \mathbb{B}_{e}$ ) such that

$$
i_{\mathrm{NL}}\left(s_{0}\right)=\frac{\sqrt{\int_{\mathbb{B}_{a} \backslash \mathbb{B}_{e}}\left(\widehat{w}\left(2 \pi v ; s_{0}\right)\right)^{2} d v}}{\sqrt{\int_{\mathbb{B}_{e}}\left(\widehat{w}\left(2 \pi v ; s_{0}\right)\right)^{2} d v}} .
$$

Figure 12 displays the graph of function $s_{0} \mapsto i_{\mathrm{NL}}\left(s_{0}\right)$. It can be seen that geometric nonlinear effects appear for $s_{0}>0.10$. Figure 13 displays the graph of function $v \mapsto\left\|\widetilde{\mathbf{u}}^{j_{0}}(2 \pi v)\right\|$ constructed with the NL-ROMF for $s_{0}$ equal to 0.04 (response belonging to the quasi-linear regime of the nonlinear response), and equal to $0.25,1.11$, and 4.0 (response belonging to the nonlinear regime of the nonlinear response). The left top figure clearly shows a dynamic response that remains in the linear regime (there is no response outside $\mathbb{B}_{e}$ ). On the other hand, subsequent contributions with unexpected resonances appear outside $\mathbb{B}_{e}$ in the frequency band $\mathbb{B}_{a} \backslash \mathbb{B}_{e}$ as soon as $s_{0}$ increases.

5.2. Linear and nonlinear dynamic analyses in the time domain using the L-ROM and NL-ROM

In this section, the linear and nonlinear dynamic responses of the tuned configuration (pattern $\mathcal{P}_{0}=24 A$ ) and three detuned configurations (patterns $\mathcal{P}_{6}=(4 A 2 B)_{4}, \mathcal{P}_{11}=B 4 A B 18 A, \mathcal{P}_{25}=$ 


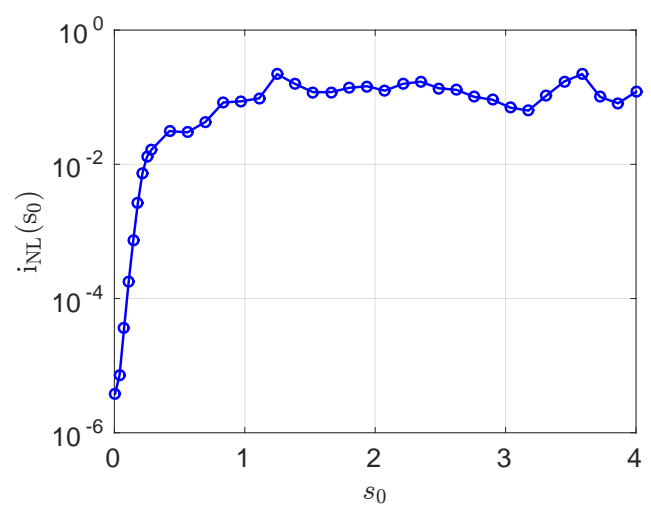

Figure 12: Sensitivity analysis with respect to parameter $s_{0}$ using the NL-ROMF for the nonlinear tuned rotating bladed disk (pattern $\mathcal{P}_{0}=24 A$ ): graph of function $s_{0} \mapsto i_{\mathrm{NL}}\left(s_{0}\right)$.
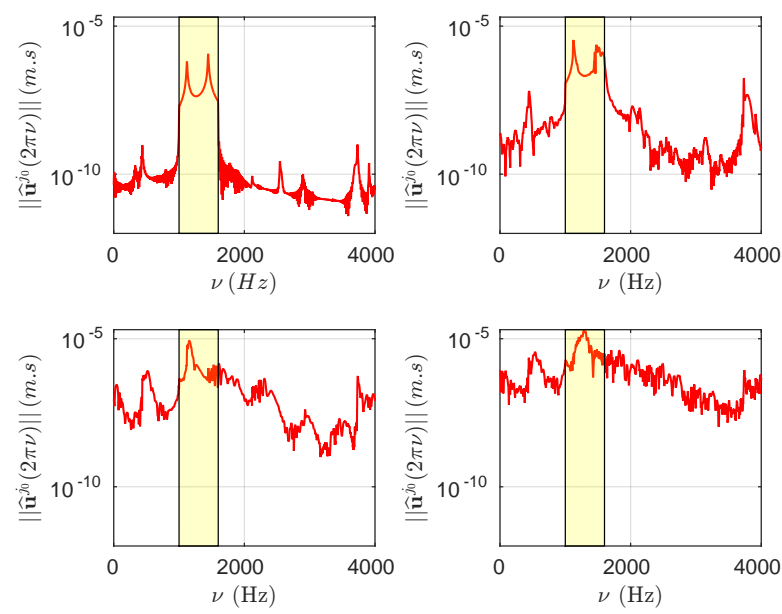

Figure 13: Sensitivity analysis with respect to parameter $s_{0}$ of the response computed with the NL-ROMF and analyzed in the frequency domain: graphs of function $v \mapsto\left\|\widehat{\mathbf{u}}^{j_{0}}(2 \pi v)\right\|$ for $s_{0}=$ 0.04 (left top figure), $s_{0}=0.25$ (right top figure), $s_{0}=1.11$ (left down figure), and $s_{0}=4.0$ (right down figure). The light yellow zone corresponds to the excitation frequency band $\mathbb{B}_{e}$

$3 A 3 B 3 A 15 B)$ are analyzed. Figure 14 displays the graph of function $t \mapsto u_{2, \mathrm{~L}}^{j_{0}}(t)$ for patterns $\left(\mathcal{P}_{0}, \mathcal{P}_{6}, \mathcal{P}_{11}, \mathcal{P}_{25}\right)$ corresponding to a linear computation performed with the L-ROM defined in Section 2.4.2 Figure 15 displays the graph of $t \mapsto u_{2, \mathrm{NL}}^{j_{0}}(t)$ for patterns $\left(\mathcal{P}_{0}, \mathcal{P}_{6}, \mathcal{P}_{11}, \mathcal{P}_{25}\right)$ corresponding to the nonlinear computation performed with the NL-ROM. By comparing the nonlinear results with the linear ones, it can be seen that $s_{0}=1$ yields high nonlinear geometric effects that mitigate the amplitude of the responses and show "irregular" responses, suggesting 
numerous resonances contributing outside band $\mathbb{B}_{e}$.
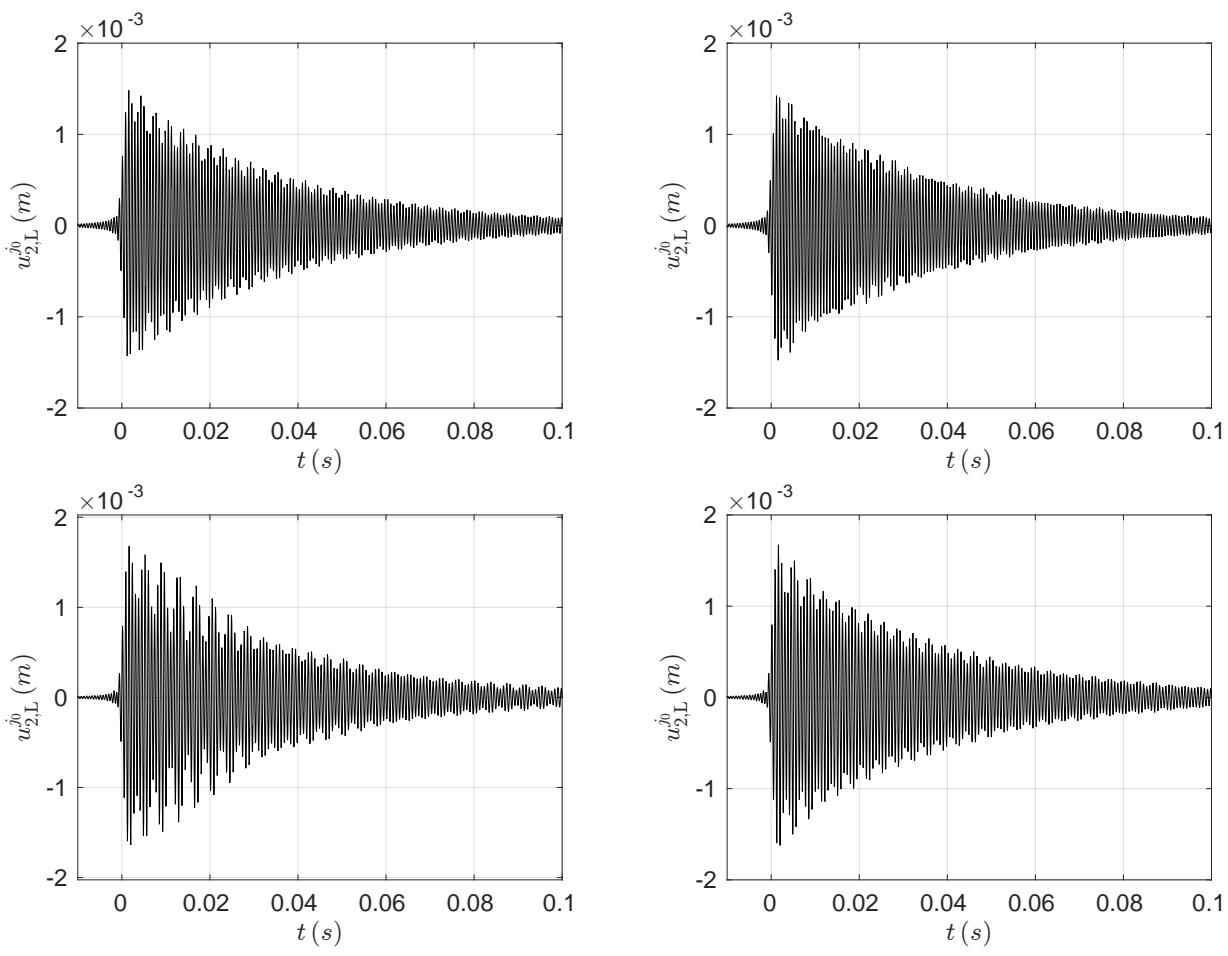

Figure 14: Zoom on the time interval $[-0.01,0.1] s$ of the linear dynamic analysis in the time domain performed with the L-ROM: graph of function $t \mapsto u_{2, \mathrm{~L}}^{j_{0}}(t)$ defined on the time interval $[-0.05,1.5] s$ for the patterns $\mathcal{P}_{0}$ (left top figure), $\mathcal{P}_{6}$ (right top), $\mathcal{P}_{11}$ (left down), and $\mathcal{P}_{25}$ (right down).

\subsection{Analysis the nonlinear dynamic time responses in the frequency domain}

A Fourier transform is then performed on the time responses constructed with the L-ROM (linear) and the NL-ROM (nonlinear), allowing the spectrum of the responses to be analyzed in the frequency band of analysis $\mathbb{B}_{a}=[0,4000] \mathrm{Hz}$. We are interested in the dynamic amplification factor $b(2 \pi v)$ (defined by Eq. (36) with respect to the tuned configuration. Figure 16 displays the graphs of functions $v \mapsto b_{\mathrm{L}}(2 \pi v)$ (linear) and $v \mapsto b_{\mathrm{NL}}(2 \pi v)$ (nonlinear) for the tuned rotating bladed disk (pattern $\mathcal{P}_{0}$ ) and for the detuned rotating bladed disks (patterns $\mathcal{P}_{6}, \mathcal{P}_{11}, \mathcal{P}_{25}$ ). By comparing the linear responses with the nonlinear ones, it can be seen the strong effects of the nonlinearities outside the frequency band of excitation $\mathbb{B}_{e}$ and that new resonances occur below and above this frequency band $\mathbb{B}_{e}$. Such phenomena has previously been observed [30, 49] in the turbomachinery context. In order to better understand the discrepancy of the detuned nonlinear dynamic responses with respect to the tuned ones, let $b_{\mathrm{NL}}^{ \pm}(2 \pi v)$ be the upper $(+)$ and the lower $(-)$ envelopes of the dynamic amplification factors. Figure 17 displays the graphs of functions $v \mapsto b_{\mathrm{NL}}^{ \pm}(2 \pi v)$ and $v \mapsto b_{\mathrm{NL}}^{\text {tuned }}(2 \pi v)$ corresponding to the nonlinear computations performed 

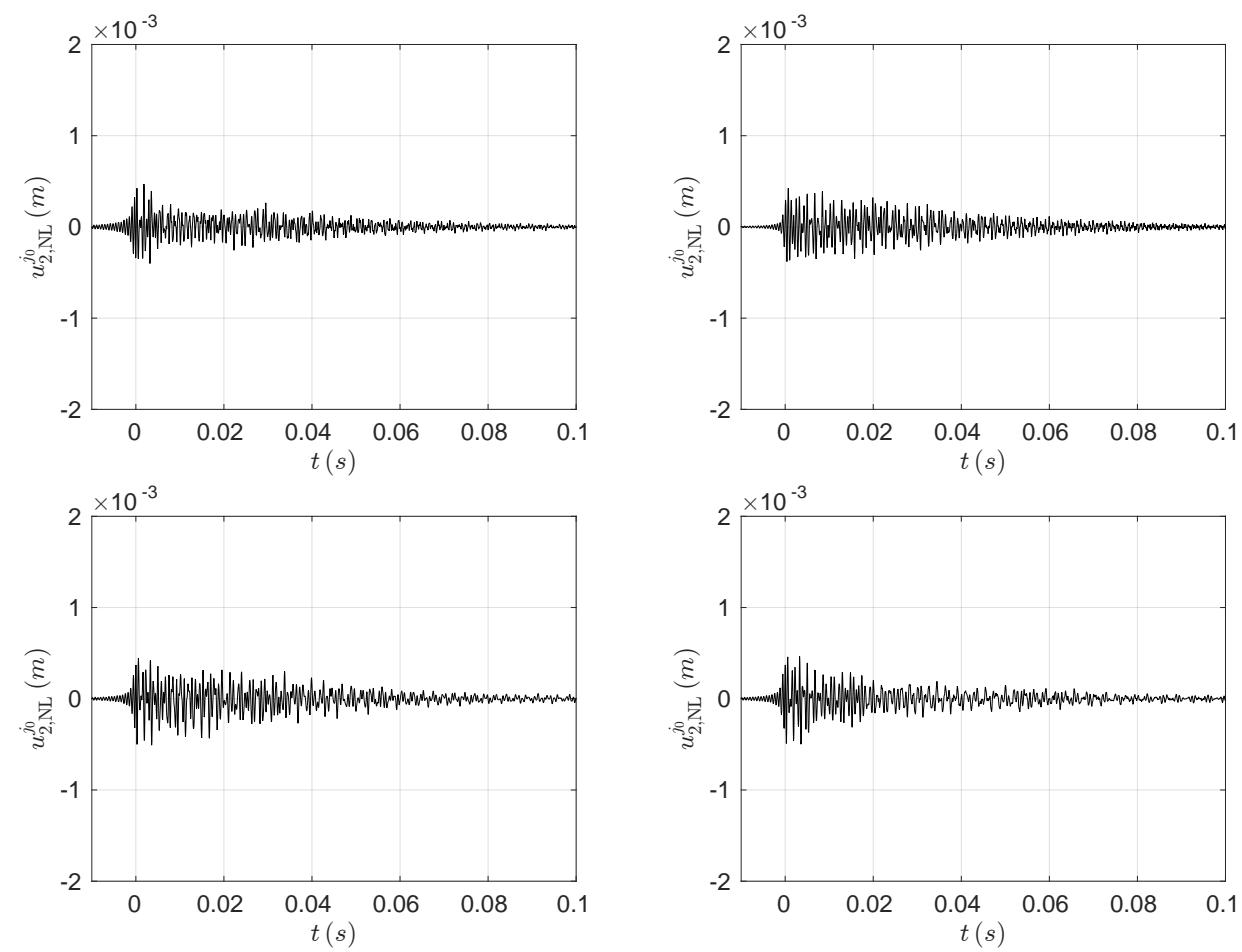

Figure 15: Zoom on the time interval $[-0.01,0.1] s$ of the nonlinear dynamic analysis in the time domain performed with the NL-ROM: graph of function $t \mapsto u_{2, \mathrm{NL}}^{j_{0}}(t)$ defined on the time interval $[-0.05,1.5] s$ for the patterns $\mathcal{P}_{0}$ (left top figure), $\mathcal{P}_{6}$ (right top), $\mathcal{P}_{11}$ (left down), and $\mathcal{P}_{25}$ (right down).

with the NL-ROM. It can be seen that the nonlinear dynamic response is very sensitive to the detuning, especially outside band $\mathbb{B}_{e}$. At a given frequency, the amplification factor can strongly differ from one pattern to another one.

\section{Analysis of the stochastic linear and nonlinear tuned and detuned rotating bladed disks with mistuning}

In all this section we consider the nonlinear tuned rotating bladed disk and the nonlinear detuned one in presence of mistuning, which are analyzed using the NL-SROM1, NL-SROM2, and L-SROM defined in Section 3

\subsection{Sensitivity analysis with respect to parameter $s_{0}$ for the tuned and detuned rotating bladed} disks in presence of mistuning

The objective is to quantify and to give explanations concerning the effects of the level of uncertainties related to the level of mistuning. In that sense, a parametric analysis is carried out with respect to (i) the dispersion parameter $\delta_{K}$ that controls the level of uncertainties in 

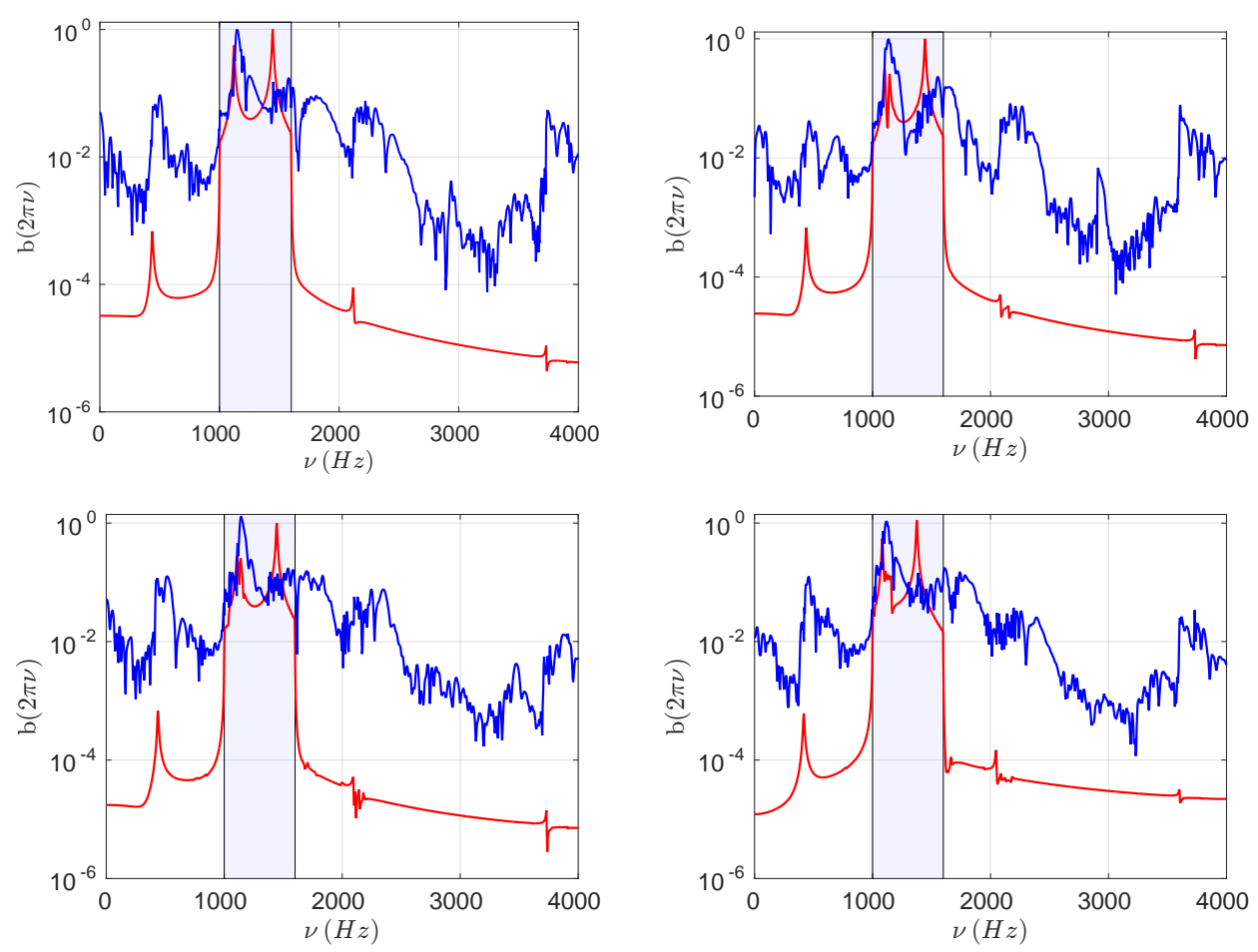

Figure 16: Frequency analysis of the time responses computed with the NL-ROM: graphs of functions $v \mapsto b_{\mathrm{L}}(2 \pi v)$ (red smooth thin lines) and $v \mapsto b_{\mathrm{NL}}(2 \pi v)$ (blue irregular thick lines) for patterns $\mathcal{P}_{0}$ (left top figure), $\mathcal{P}_{6}$ (right top), $\mathcal{P}_{11}$ (left down), and $\mathcal{P}_{25}$ (right down). The excitation frequency band $\mathbb{B}_{e}$ is in light grey area.

the computational model and (ii) parameter $s_{0}$. A comparison is performed between the linear stochastic responses computed with L-SROM and the nonlinear stochastic responses computed with NL-SROM2. Let $\widehat{U}_{\max }\left(s_{0}\right)$ be the real-valued random variable defined by $\widehat{U}_{\max }\left(s_{0}\right)=$ $\max _{v \in \mathbb{B}_{e}}\left\|\mathbf{U}^{j_{0}}\left(2 \pi v ; s_{0}\right)\right\|$ depending on $s_{0}$. Let $\widehat{u}_{\text {max }}\left(s_{0}\right)$ be the real number depending on $s_{0}$ such that Proba $\left\{\widehat{\mathrm{U}}_{\max }\left(\mathrm{s}_{0}\right) \leq \widehat{\mathrm{u}}_{\max }\left(\mathrm{s}_{0}\right)\right\} \leq 0.95$. Figure 18 displays the function $s_{0} \mapsto \widehat{u}_{\max }\left(s_{0}\right)$ computed with the stochastic models L-SROM and NL-SROM2 for $\delta_{K}=0.1$ and for the patterns $\mathcal{P}_{0}$ (tuned) and $\mathcal{P}_{6}$ (detuned). It can be seen that the propagation of uncertainties for the nonlinear geometrical effects (NL-SROM2) is smaller than for the linear case (L-SROM). This attenuation is more important while $s_{0}$ is increasing, that is to say when the nonlinear effects increase. Moreover, Figure 19 displays the graph of the confidence region of $v \mapsto\left\|\widehat{\mathbf{U}}^{j_{0}}(2 \pi v)\right\|$ corresponding to a probability level 0.95 for both patterns $\mathcal{P}_{0}$ (tuned) and $\mathcal{P}_{6}$ (detuned) computed using NL-SROM2 for three external-forces intensities $s_{0}=0.01, s_{0}=0.15$, and $s_{0}=1$ corresponding to negligible, moderate, and large geometric nonlinear effects. It can be seen that for $s_{0}=0.01$, the response in the frequency domain is clearly located in excitation frequency band $\mathbb{B}_{e}$, similarly to the linear case. For the medium and high values of $s_{0}$, geometric nonlinear effects yield unexpected resonances that occur outside $\mathbb{B}_{e}$, especially, around $484 \mathrm{~Hz}$ (mode 1 defined in Section 4.3), which 
corresponds to the first bending mode of the blade and $3700 \mathrm{~Hz}$, which corresponds to a combination of elastic modes. In addition, the general level of responses outside band $\mathbb{B}_{e}$ increase with $s_{0}$. Concerning frequency band of excitation $\mathbb{B}_{e}$, it can be seen that the second bending mode of blade (mode 2 around $1170 \mathrm{~Hz}$ defined in Section 4.3 ) is relatively stable in frequency with respect to $s_{0}$ while the first torsion mode of blade (mode 3 at around $1490 \mathrm{~Hz}$ defined in Section 4.3 tends to vanish when the nonlinear geometric effects increase. Furthermore, it can be noticed that the width of the confidence region is not constant with respect to the frequency. Let $B_{\mathrm{L}}\left(2 \pi v ; \delta_{K}\right)$ be the random variable depending on $\delta_{K}$, defined by Eq. (37), and constructed using the L-SROM. Let $B_{\mathrm{L}}^{\infty}\left(\delta_{K}\right)$ be the random variable defined by

$$
B_{\mathrm{L}}^{\infty}\left(\delta_{K}\right)=\max _{v \in \mathbb{B}_{e}} B_{\mathrm{L}}\left(2 \pi v, \delta_{K}\right)
$$

which corresponds to the maximum dynamic amplification factor over the excitation frequency band. We then denote by $b_{\mathrm{L}}^{+, \infty}\left(\delta_{K}\right)$ the value of $B_{\mathrm{L}}^{\infty}\left(\delta_{K}\right)$ depending on $\delta_{K}$ and such that

$$
\operatorname{Proba}\left\{\mathrm{B}_{\mathrm{L}}^{\infty}\left(\delta_{\mathrm{K}}\right) \leq \mathrm{b}_{\mathrm{L}}^{+, \infty}\left(\delta_{\mathrm{K}}\right)\right\} \leq 0.95 .
$$

Figure 20 displays the graph of function $\delta_{K} \mapsto b_{\mathrm{L}}^{+, \infty}\left(\delta_{K}\right)$ for seven patterns of configurations: tuned pattern $\mathcal{P}_{0}$ and detuned ones $\mathcal{P}_{2}, \mathcal{P}_{3}, \mathcal{P}_{5}, \mathcal{P}_{6}, \mathcal{P}_{12}$, and $\mathcal{P}_{31}$ defined in Appendix A. The results obtained are coherent with those published in [25], especially, for each pattern, a maximum is obtained for a small mistuning (small value of $\delta_{K}$ ).

\subsection{Stochastic analysis of nonlinear tuned and detuned rotating bladed disks in presence of mistuning for patterns}

In this section, we present the results obtained using NL-SROM1 for the nonlinear tuned and detuned rotating bladed disks in presence of mistuning.

Let $B_{\mathrm{NL}}\left(2 \pi v ; \delta_{K}\right)$ be the random variable depending on $\delta_{K}$, defined by Eq. (37), and constructed using the NL-SROM1. For two values of $\delta_{K}$ controlling the mistuning level, Figure $21\left(\delta_{K}=\right.$ $0.03)$ and Figure $22\left(\delta_{K}=0.1\right)$ display the confidence region of random variable $B_{\mathrm{NL}}\left(2 \pi v ; \delta_{K}\right)$, estimated with a probability level of 0.95 , for configurations $\mathcal{P}_{0}$ (tuned), and for $\mathcal{P}_{6}, \mathcal{P}_{11}$, and $\mathcal{P}_{25}$ (detuned defined in Appendix A). These figures allow for estimating the robustness of the responses with respect to the level of uncertainties as a function of the considered patterns. Nevertheless, the first torsion mode for $h=4$ (mode 3 around $1490 \mathrm{~Hz}$ defined in Section 4.3 located in $\mathbb{B}_{e}$ is very sensitive to the mistuning, as already mentioned for the linear case in Section 6.1 It can be seen that the nonlinear stochastic response of the mistuned-detuned bladed disk is particularly complex. It should also be noted, as in Section 6.1, that unexpected resonances occur outside the excitation frequency band as soon as the level of nonlinearities is significant.

\subsection{Stochastic analysis of nonlinear tuned and detuned rotating bladed disks in presence of mistuning for 46 patterns}

The analysis that we have presented in Section 6.2 is revisited considering all the 46 patterns defined in Appendix A. For simplifying the presentation of the results, the 46 patterns (the tuned pattern and the 45 detuned patterns, all in presence of mistuning) are considered as 46 realizations of a random mechanical system. Let $B_{\mathrm{NL}}^{\text {all }}(2 \pi v)$ be the random amplification factor defined by Eq. (37) of this random mechanical system, estimated using the NL-SROM1 for $\delta_{K}=0.1$. In practice, the confidence region associated with a probability level of 0.95 of random variable $B_{\mathrm{NL}}^{\text {all }}(2 \pi v)$ is estimated in concatenating all the Monte-Carlo realizations computed for each one 
of the 46 patterns. Figure 23 displays (in linear and log scales) the confidence region of the deterministic amplification factor $b_{\mathrm{NL}}^{\text {tuned }}(2 \pi v)$ for the tuned rotating bladed disk without mistuning and the random variable $B_{\mathrm{NL}}^{\text {all }}(2 \pi v)$ for all the 46 patterns defined in Appendix A, which includes, as previously mentioned, the tuned pattern $\mathcal{P}_{0}$ in presence of mistuning. We use the same type of analysis as the one that we have presented in Section 6.2. This figure shows that the values of the random amplification factor, which occur outside excitation frequency band $\mathbb{B}_{e}$ is significant and is sensitive to uncertainties. It should be noted that $b_{\mathrm{NL}}^{\text {tuned }}(2 \pi v)$ is included in the confidence region. Moreover, the analysis of Figure 23 shows that the robustness of the stochastic response around the two main resonances located in band $\mathbb{B}_{e}$ is significantly higher than outside $\mathbb{B}_{e}$, while noting that there are relatively high levels outside $\mathbb{B}_{e}$ (in linear, there is no response outside the band $\mathbb{B}_{e}$ ). In the low-frequency band $[0,1000] \mathrm{Hz}$ (not excited by the external forces), there are mistuned configurations for which the amplitude level outside $\mathbb{B}_{e}$ is four times lower than the one in $\mathbb{B}_{e}$. Nevertheless, it should be noted that the levels of responses (induced by the nonlinear geometric effects), which occur outside band $\mathbb{B}_{e}$, depend on the bladed disk, and that these levels could be larger than in the frequency band $\mathbb{B}_{e}$ for other bladed disks.

These results lead us to split the frequency band of analysis $\mathbb{B}_{a}$ in 3 sub-frequency bands to better analyze the amplification factor. We then define the following bands: $\mathbb{B}_{\text {low }}=[0,1000] \mathrm{Hz}$, $\mathbb{B}_{\text {med }}=[1000,1300] \mathrm{Hz}$, and $\mathbb{B}_{\text {high }}=[1300,4000] \mathrm{Hz}$. Note that band $\mathbb{B}_{\text {med }}$ is included in frequency band of excitation $\mathbb{B}_{e}$ and that band $\mathbb{B}_{\text {high }}$ overlaps band $\mathbb{B}_{e}$ with the common frequency band $[1300,1600] \mathrm{Hz}$. This partition of the frequency band of analysis has been introduced in order to analyze the amplification of the resonances in each sub-frequency band. Let $\{$ low, med, high $\}$ be the set of the three strings of characters such that, for band $\in\{$ low, med, high $\}$, the band $\mathbb{B}_{\text {band }}$ denotes one of the band $\mathbb{B}_{\text {low }}, \mathbb{B}_{\text {med }}$, and $\mathbb{B}_{\text {high }}$.

Let $B_{\mathrm{NL}}\left(2 \pi v ; \delta_{K}\right)$ be the random amplification factor defined by Eq. [37], computed using NL-SROM1. Let

$$
B_{\mathrm{NL}}^{\infty, \text { band }}\left(\delta_{K}\right)=\max _{v \in \mathbb{B}_{\text {band }}} B_{\mathrm{NL}}\left(2 \pi v ; \delta_{K}\right)
$$

be the random variable that corresponds to the maximum dynamic amplification factor over frequency band $\mathbb{B}_{\text {band }}$. We then denote by $b_{\mathrm{NL}}^{+, \infty \text {,band }}\left(\delta_{K}\right)$ the value of $B_{\mathrm{NL}}^{\infty, \text { band }}\left(\delta_{K}\right)$ depending on $\delta_{K}$ and such that

$$
\operatorname{Proba}\left\{\mathrm{B}_{\mathrm{NL}}^{\infty, \text { band }}\left(\delta_{\mathrm{K}}\right) \leq \mathrm{b}_{\mathrm{NL}}^{+, \infty, \text { band }}\left(\delta_{\mathrm{K}}\right)\right\} \leq 0.95 .
$$

For anyone of the 46 patterns, we are interested in plotting the graphs of functions $\delta_{K} \mapsto$ $b_{\mathrm{NL}}^{+, \infty \text {,med }}\left(\delta_{K}\right), \delta_{K} \mapsto b_{\mathrm{NL}}^{+, \infty, \text { low }}\left(\delta_{K}\right)$, and $\delta_{K} \mapsto b_{\mathrm{NL}}^{+, \infty, \text { high }}\left(\delta_{K}\right)$, which describe the evolution of the maximum amplification factor for each configuration according to the dispersion parameter $\delta_{K}$. However, to maintain a sufficient readability of the figures, we only plot the lower and upper envelopes of the 46 configurations. These two envelopes define a region in which all the 46 configurations belong. Figures 24, 25, and 26 show the graphs for each frequency band, $\mathbb{B}_{\text {med }}$, $\mathbb{B}_{\text {low }}$, or $\mathbb{B}_{\text {high. }}$ In the caption of each one of these three figures, the patterns corresponding to the lower and upper envelopes will be indicated. Figure 24 shows that there is a weak sensitivity of the envelopes with respect to the mistuning level represented by the value of $\delta_{K}$. Pattern $\mathcal{P}_{34}$, which corresponds to the upper envelope, yields the largest dynamic amplification factor in band $\mathbb{B}_{\text {med }}$, whereas pattern $\mathcal{P}_{1}$, which corresponds to the lower envelope, has the lowest dynamic amplification factor. In Figures 25 and 26 it can be seen that the envelopes are sensitive to the level of mistuning represented by $\delta_{K}$, and that a very high dynamic amplification factor can be obtained, for instance pattern $\mathcal{P}_{26}$ (upper envelope for $\mathbb{B}_{\text {low }}$ ) and pattern $\mathcal{P}_{9}$ (upper envelope for $\mathbb{B}_{\text {high }}$ ). Note that these high dynamic amplification factors are due to the choice of the reference. 
If this reference was chosen as the linear tuned system, then this amplification dynamic factor would be infinite. Presently, the reference has been chosen as the nonlinear tuned system without mistuning.

\section{CONCLUSION}

We have presented a robust analysis of the effects of geometric nonlinearities on the nonlinear dynamic behavior of rotating bladed disks that are detuned in presence of mistuning. This mistuning induces uncertainties that are taken into account by a probabilistic approach in the computational model. The results obtained allow for increasing the knowledge in the area of the nonlinear stochastic dynamic of the detuned rotating bladed disks. It has been demonstrated that the responses obtained in the frequency band outside the band of excitation can be significant. The envelopes of the dynamic amplifications factors among the investigated patterns show that the nonlinear dynamic response is sensitive to the detuning in presence of mistuning. The results highlight the indirect excitation of the rotating bladed disks through the geometric nonlinearities outside the excitation frequency band. The optimization with respect to all the possible configurations defined by the patterns, with the objective to find the pattern that minimizes the random dynamic amplification factor, remains a problem that demands large computer resources in term of CPU time. The complexity of the results obtained for the 46 configurations studied, seems to show that such a discrete nonconvex optimization problem on a set of configurations having a huge number of patterns, is difficult. Nevertheless, although a nonexhaustive study optimization could not be made, we have shown that there were detuned configurations that minimize the dynamic amplification factor in presence of mistuning. 


\section{A. Table of patterns}

\begin{tabular}{|c|c|}
\hline Pattern number & Arrangement \\
\hline $\mathcal{P}_{0}$ & $24 A$ \\
\hline $\mathcal{P}_{1}$ & $(5 A 1 B)_{4}$ \\
\hline $\mathcal{P}_{2}$ & $(A B)_{12}$ \\
\hline $\mathcal{P}_{3}$ & $(4 A 4 B)_{3}$ \\
\hline $\mathcal{P}_{4}$ & $4 A 2 B 3 A 2 B 5 A 2 B 3 A 2 B$ \\
\hline $\mathcal{P}_{5}$ & $(3 A 3 B)_{4}$ \\
\hline $\mathcal{P}_{6}$ & $(4 A 2 B)_{4}$ \\
\hline $\mathcal{P}_{7}$ & $A B 2 A 2 B(A B)_{2} 2 A 2 B 2 A A B 2 B(A B)_{2}$ \\
\hline $\mathcal{P}_{8}$ & $2 A B A 2 B 2 A 3 B(A B)_{2} 2 A B 3 A 3 B$ \\
\hline $\mathcal{P}_{9}$ & $(2 A 2 B)_{6}$ \\
\hline $\mathcal{P}_{10}$ & $4 A 4 B(2 A 2 B)_{2} 2 A 6 B$ \\
\hline $\mathcal{P}_{11}$ & $B 4 A B 18 A$ \\
\hline $\mathcal{P}_{12}$ & $12 A 12 B$ \\
\hline $\mathcal{P}_{13}$ & $6 B 12 A 3 B 3 A$ \\
\hline $\mathcal{P}_{14}$ & $3 B 15 A 3 B 3 A$ \\
\hline $\mathcal{P}_{15}$ & $6 A 3 B 6 A 9 B$ \\
\hline $\mathcal{P}_{16}$ & $(3 B 6 A)_{2} 3 B 3 A$ \\
\hline $\mathcal{P}_{17}$ & $3 A 6 B 3 A 12 B$ \\
\hline $\mathcal{P}_{18}$ & $3 B 12 A 6 B 3 A$ \\
\hline $\mathcal{P}_{19}$ & $18 A 6 B$ \\
\hline $\mathcal{P}_{20}$ & $3 B 12 A 6 B 3 A$ \\
\hline $\mathcal{P}_{21}$ & $6 B 9 A 6 B 3 A$ \\
\hline $\mathcal{P}_{22}$ & $6 A 3 B 3 A 12 B$ \\
\hline $\mathcal{P}_{23}$ & $9 A 3 B 6 A 6 B$ \\
\hline $\mathcal{P}_{24}$ & $14 A 9 B$ \\
\hline $\mathcal{P}_{25}$ & $3 A 3 B 3 A 15 B$ \\
\hline $\mathcal{P}_{26}$ & $15 B 9 A$ \\
\hline $\mathcal{P}_{27}$ & $3 B 6 A 12 B 3 A$ \\
\hline $\mathcal{P}_{28}$ & $3 A 21 B$ \\
\hline $\mathcal{P}_{29}$ & $3 A 3 B(3 A 6 B)_{2}$ \\
\hline $\mathcal{P}_{30}$ & $(3 A 3 B)_{2} 3 A 9 B$ \\
\hline $\mathcal{P}_{31}$ & $(6 A 6 B)_{2}$ \\
\hline $\mathcal{P}_{32}$ & $3 B 9 A 9 B 3 A$ \\
\hline $\mathcal{P}_{33}$ & $3 B 21 A$ \\
\hline $\mathcal{P}_{34}$ & $6 A 6 B 3 A 9 B$ \\
\hline $\mathcal{P}_{35}$ & $18 A 6 B$ \\
\hline $\mathcal{P}_{36}$ & $3 B 12 A 3 B 6 A$ \\
\hline $\mathcal{P}_{37}$ & $3 B 6 A 3 B 3 A 6 B 3 A$ \\
\hline $\mathcal{P}_{38}$ & $6 A 8 B 3 A 6 B$ \\
\hline $\mathcal{P}_{39}$ & $9 A 3 B 3 A 9 B$ \\
\hline $\mathcal{P}_{40}$ & $3 B 9 A 3 B 3 A 3 B 3 A$ \\
\hline $\mathcal{P}_{41}$ & $3 B 6 A 6 B 3 A 3 B 3 A$ \\
\hline $\mathcal{P}_{42}$ & $3 B 9 A 6 B 6 A$ \\
\hline $\mathcal{P}_{43}$ & $27 \quad(3 A 3 B)_{4}$ \\
\hline $\mathcal{P}_{44}$ & $(3 A 9 B)_{2}$ \\
\hline $\mathcal{P}_{45}$ & $(9 A 3 B)_{2}$ \\
\hline
\end{tabular}




\section{References}

[1] S. Tobias, R. Arnold, The influence of dynamical imperfection on the vibration of rotating disks, Proceedings of the Institution of Mechanical Engineers 171 (1) (1957) 669-690.

[2] D. Ewins, The effects of detuning upon the forced vibrations of bladed disks, Journal of Sound and Vibration 9 (1) (1969) 65-79.

[3] D. Whitehead, The maximum factor by which forced vibration of blades can increase due to mistuning, ASME Journal of Engineering for Gas Turbines and Power 120 (1) (1998) 115-119.

[4] M. Kruse, C. Pierre, Forced response of mistuned bladed disks using reduced-order modeling, in: 37th Structure, Structural Dynamics and Materials Conference, 1996, p. 1545.

[5] M.-T. Yang, J. Griffin, A reduced order approach for the vibration of mistuned bladed disk assemblies, ASME Journal of Engineering for Gas Turbines and Power 119 (1) (1997) 161-167.

[6] M. Castanier, C. Pierre, Consideration on the benefits of intentional blade mistuning for the forced response of turbomachinery rotors, Analysis and design issues for modern aerospace vehicles (1997) 419-425.

[7] R. Bladh, M. Castanier, C. Pierre, Reduced order modeling and vibration analysis of mistuned bladed disk assemblies with shrouds, Journal of engineering for Gas Turbines and Power 121 (3) (1999) 515-522.

[8] R. Bladh, M. Castanier, Component-mode-based reduced order modeling techniques for mistuned bladed disks-part 1: Theoretical models, Journal of Engineering for Gas Turbines and Power 123 (1) (2001) 89-99.

[9] A. Rivas-Guerras, M. Mignolet, Local/global effects of mistuning on the forced response of bladed disks, in: ASME Turbo Expo 2001: Power for Land, Sea, and Air, American Society of Mechanical Engineers, 2001, pp. V004T01A002-V004T01A002.

[10] M. Castanier, C. Pierre, Using intentional mistuning in the design of turbomachinery rotors, AIAA journal 40 (10) (2002) 2077-2086.

[11] D. Feiner, J. Griffin, A fundamental model of mistuning for a single family of modes, ASME Journal of Turbomachinery 124 (4) (2002) 597-605.

[12] J. Kenyon, J. Griffin, D. Feiner, Maximum bladed disk forced response from distortion of a structural mode, Journal of Turbomachinery 125 (2) (2003) 352-363.

[13] S.-H. Lim, R. Bladh, M. Castanier, C. Pierre, Compact, generalized component mode mistuning representation for modeling bladed disk vibration, AIAA journal 45 (9) (2007) 2285-2298.

[14] B. Beirow, F. Figaschewsky, A. Kühhorn, A. Bornhorn, Vibration analysis of an axial turbine blisk with optimized intentional mistuning pattern, Journal of Sound and Vibration 442 (2019) 11-27.

[15] M. Castanier, C. Pierre, Investigation of the combined effects of intentional and random mistuning on the forced response of bladed disks, in: 34th AIAA/ASME/SAE/ASEE Joint Propulsion Conference and Exhibit, 1998, p. 3720.

[16] M. Mignolet, W. Hu, I. Jadic, On the forced response of harmonically and partially mistuned bladed disks. part 2: partial mistuning and applications, International Journal of Rotating Machinery 6 (1) (2000) 43-56.

[17] M. Mignolet, A. Rivas-Guerra, J. Delor, Identification of mistuning characteristics of bladed disks from free response data -part 1, Journal of Engineering for Gas Turbines and Power 123 (2) (2001) 395-403.

[18] J. M. Brown, J. Slater, R. Grandhi, Probabilistic analysis of geometric uncertainty effects on blade modal response, in: ASME Turbo Expo 2003, collocated with the 2003 International Joint Power Generation Conference, American Society of Mechanical Engineers, 2003, pp. 247-255.

[19] S.-Y. Lee, M. Castanier, C. Pierre, Assessment of probabilistic methods for mistuned bladed disk vibration, in: 46th AIAA/ASME/ASCE/AHS/ASC Structures, Structural Dynamics and Materials Conference, 2005, p. 1990.

[20] E. Capiez-Lernout, C. Soize, Nonparametric modeling of random uncertainties for dynamic response of mistuned bladed-disks., Journal of Engineering for Gas Turbines and Power 126 (3) (2004) 610-618.

[21] E. Capiez-Lernout, C. Soize, J.-P. Lombard, C. Dupont, E. Seinturier, Blade manufacturing tolerances definition for a mistuned industrial bladed disk, ASME Journal of Engineering for Gas Turbines and Power, (In press) 127 (2).

[22] F. Nyssen, M. Arnst, J.-C. Golinval, Modeling of uncertainties in bladed disks using a nonparametric approach, in: ASME 2014 International Design Engineering Technical Conferences and Computers and Information in Engineering Conference, American Society of Mechanical Engineers, 2014, pp. V008T11A068-V008T11A068.

[23] B.-K. Choi, J. Lentz, A. Rivas-Guerra, M. Mignolet, Optimization of intentional mistuning patterns for the reduction of the forced response effects of unintentional mistuning: Formulation and assessment, in: International Gas Turbine and Aeroengine Congress and Exhibition, New Orleans, 2001.

[24] Y. Han, M. Mignolet, Optimization of intentional mistuning patterns for the mitigation of the effects of random mistuning, in: ASME Turbo Expo 2008: Power for Land, Sea, and Air, American Society of Mechanical Engineers, 2008, pp. 601-609.

[25] M. Mbaye, C. Soize, J.-P. Ousty, E. Capiez-Lernout, Robust analysis of design in vibration of turbomachines, Journal of Turbomachinery 135 (2) (2013) 021008. 
[26] Y. Han, R. Murthy, M.-P. Mignolet, J. Lentz, Optimization of intentional mistuning patterns for the mitigation of the effects of random mistuning, Journal of Engineering for Gas Turbines and Power 136 (6) (2014) 062505.

[27] A. Vakakis, Dynamics of a nonlinear periodic structure with cyclic symmetry, Acta Mechanica 95 (1-4) (1992) $197-226$.

[28] A. Grolet, F. Thouverez, Vibration analysis of a nonlinear system with cyclic symmetry, in: ASME Turbo Expo 2010: Power for Land, Sea, and Air, American Society of Mechanical Engineers, 2010, pp. 917-929.

[29] A. Martin, F. Thouverez, Dynamic analysis and reduction of a cyclic symmetric system subjected to geometric nonlinearities, Journal of Engineering for Gas Turbines and Power 141 (4) (2019) 041027.

[30] E. Capiez-Lernout, C. Soize, M. Mbaye, Mistuning analysis and uncertainty quantification of an industrial bladed disk with geometrical nonlinearity, Journal of Sound and Vibration 356 (2015) 124-143.

[31] S. Willeke, C. Keller, J. Panning-von Scheidt, J. R. Seume, J. Wallaschek, Reduced-order modeling of mistuned bladed disks considering aerodynamic coupling and mode family interaction., in: 12th European Conference on Turbomachinery Fluid Dynamics and Thermodynamics, 2017.

[32] C. Keller, A. Kellersmann, J. Friedrichs, J. R. Seume, Influence Of Geometric Imperfections On Aerodynamic And Aeroelastic Behavior Of A Compressor Blisk, in: Proceedings Of The Asme Turbo Expo: Turbine Technical Conference And Exposition, 2017, Vol 7B, 2017, ASME Turbo Expo: Turbine Technical Conference and Exposition, Charlotte, NC, JUN 26-30, 2017.

[33] C. Martel, J. J. Sanchez-Alvarez, Intentional mistuning effect in the forced response of rotors with aerodynamic damping, Journal of Sound and Vibration 433 (2018) 212-229.

[34] L. Sirovich, Turbulence and the dynamics of coherent structures. i. coherent structures, Quarterly of applied mathematics 45 (3) (1987) 561-571.

[35] P. Cizmas, B. Richardson, T. Brenner, T. OBrien, R. Breault, Acceleration techniques for reduced-order models based on proper orthogonal decomposition, Journal of Computational Physics 227 (16) (2008) 7791-7812.

[36] C. Soize, Uncertainty Quantification: An Accelerated Course with Advanced Applications in Computational Engineering, Springer, 2017.

[37] C. Desceliers, C. Soize, Nonlinear viscoelastodynamic equations of three-dimensional rotating structures in finite displacement and finite element discretization, International Journal of Nonlinear Mechanics 39 (2004) 343-368.

[38] G. Genta, Vibration of structures and machines: practical aspects, Springer Verlag, 1998.

[39] F. Ehrich, Handbook of Rotordynamics, Krieger Publishing Company, 2004.

[40] J. S. Rao, History of Rotating Machinery Dynamics, 1st Edition, History of Mechanism and Machine Science 20 , Springer Netherlands, 2011.

[41] M. Mignolet, A. Przekop, S. Rizzi, S. Spottswood, A review of indirect/non-intrusive reduced order modeling of nonlinear geometric structures, Journal of Sound and Vibration 332 (10) (2013) 2437-2460.

[42] D. Laxalde, L. Salles, L. Blanc, F. Thouverez, et al., Non-linear modal analysis for bladed disks with friction contact interfaces, in: ASME Turbo Expo 2008: Power for Land, Sea, and Air, American Society of Mechanical Engineers Digital Collection, 2008, pp. 457-467.

[43] C. Joannin, F. Thouverez, B. Chouvion, Reduced-order modelling using nonlinear modes and triple nonlinear modal synthesis, Computers \& Structures 203 (2018) 18-33.

[44] G. Golub, C. Van Loan, Matrix computations, Vol. 3, JHU press, 2012

[45] M.-P. Mignolet, C. Soize, Stochastic reduced order models for uncertain geometrically nonlinear dynamical systems, Computer Methods in Applied Mechanics and Engineering 197 (45-48) (2008) 3951-3963.

[46] E. Capiez-Lernout, C. Soize, M.-P. Mignolet, Computational stochastic statics of an uncertain curved structure with geometrical nonlinearity in three-dimensional elasticity, Computational Mechanics 49 (1) (2012) 87-97.

[47] E. Capiez-Lernout, C. Soize, An improvement of the uncertainty quantification in computational structural dynamics with nonlinear geometrical effects, International Journal for Uncertainty Quantification 7 (1) (2017) 83-98.

[48] C. Soize, A nonparametric model of random uncertainties for reduced matrix models in structural dynamics, Probabilistic Engineering Mechanics 15 (3) (2000) 277-294.

[49] A. Picou, E. Capiez-Lernout, C. Soize, M. Mbaye, Effects of geometrical nonlinearities for a rotating intentionally mistuned bladed-disk, in: Conference on Noise and Vibration Engineering (ISMA 2018), KU Leuven, 2018, pp. 1-11. 

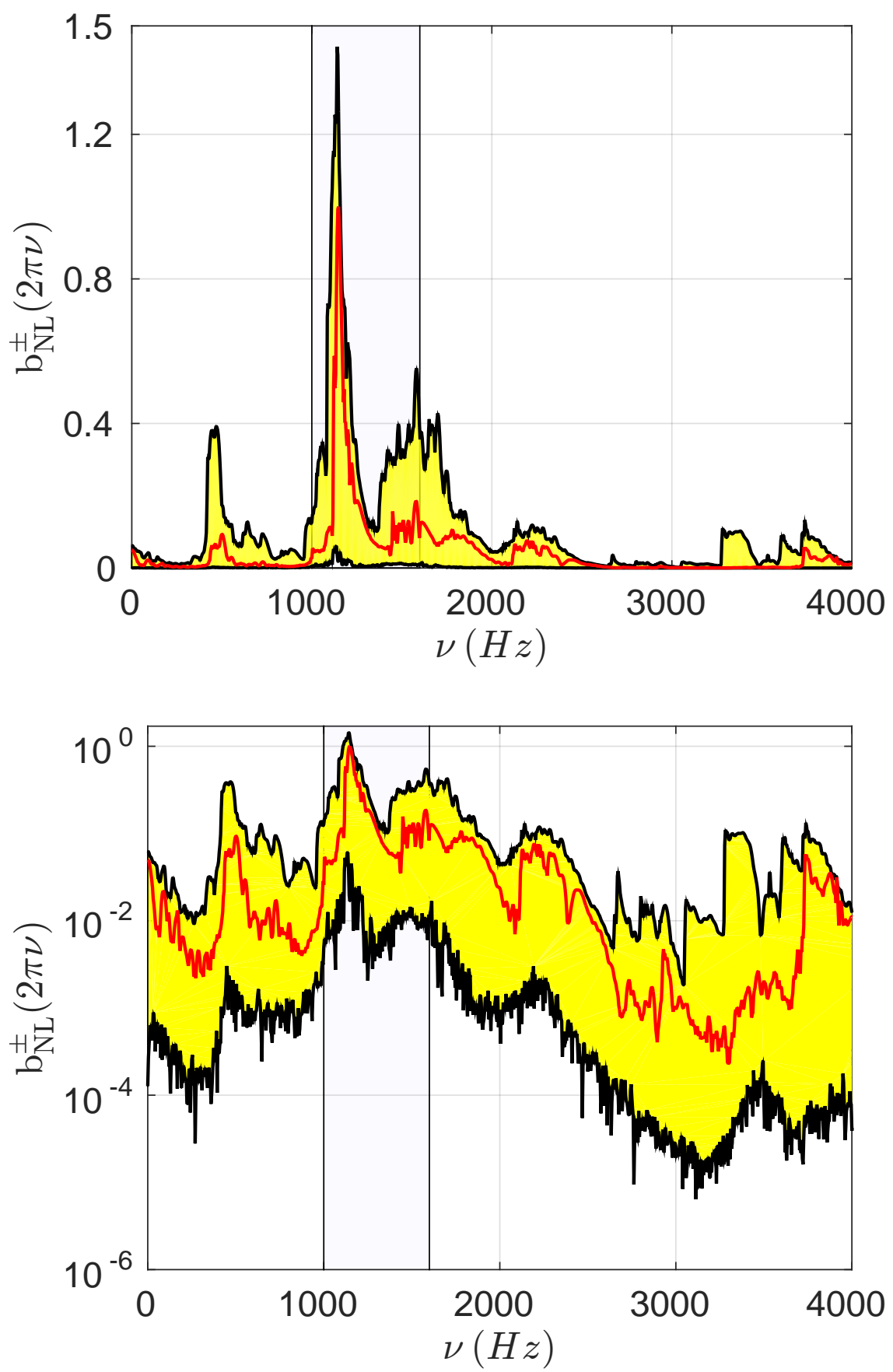

Figure 17: Graphs of functions $v \mapsto b_{\mathrm{NL}}^{ \pm}(2 \pi v)$ (black irregular thick lines) and $v \mapsto b_{\mathrm{NL}}^{\text {tuned }}(2 \pi v)$ (red/grey irregular thin line) corresponding to the upper $(+)$ and the lower $(-)$ envelopes of the dynamic amplification factor among the investigated patterns. Linear scale (top figure) and $\log$ scale (down figure) 

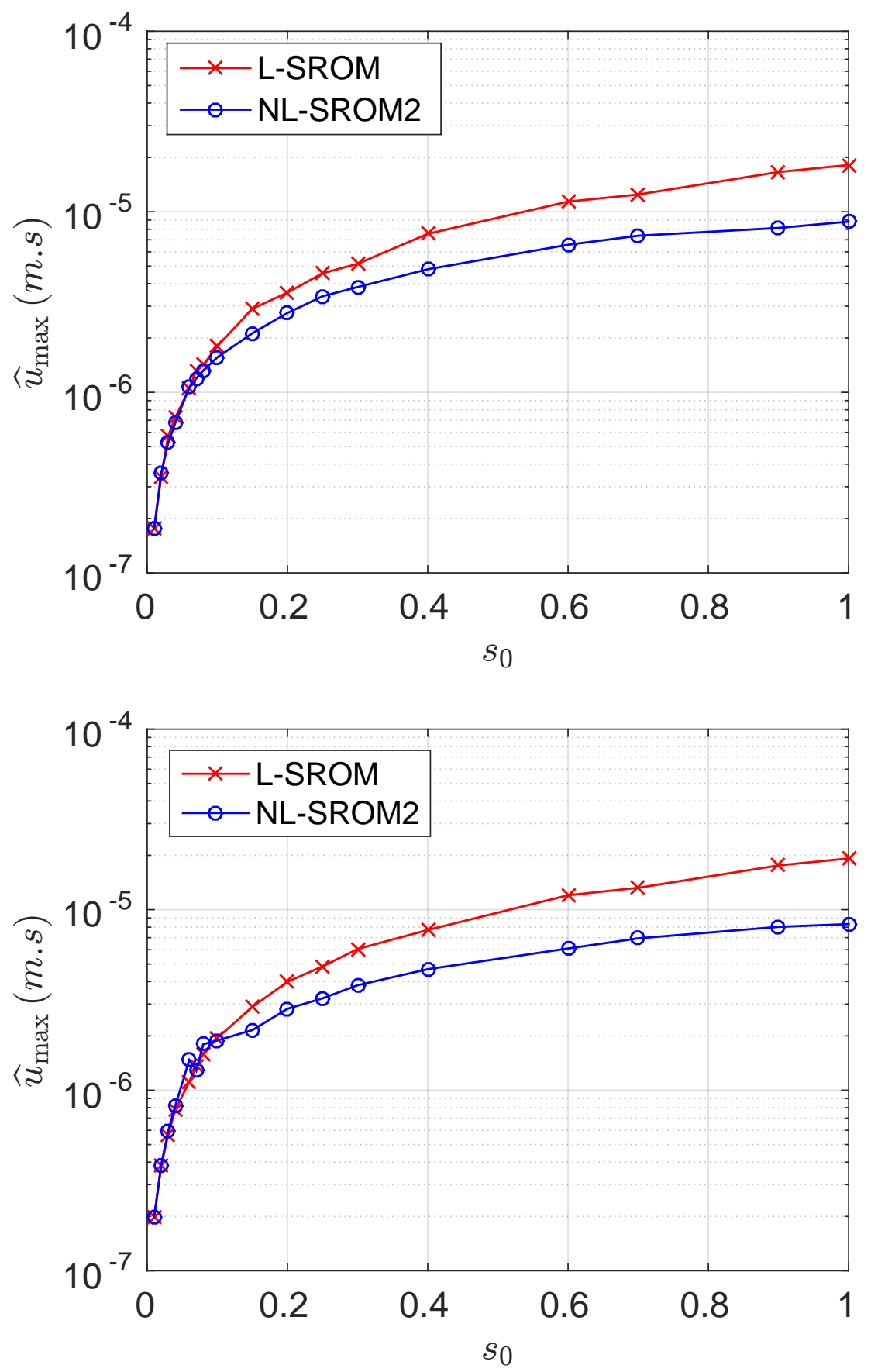

Figure 18: For $\delta_{K}=0.1$, graphs of function $s_{0} \mapsto \widehat{u}_{\max }\left(s_{0}\right)$ such that Proba $\left\{\widehat{\mathrm{U}}_{\max }\left(\mathrm{s}_{0}\right) \leq\right.$ $\left.\widehat{\mathrm{u}}_{\max }\left(\mathrm{s}_{0}\right)\right\} \leq 0.95$ : tuned rotating bladed disk $\mathcal{P}_{0}$ (top figure) and detuned rotating bladed disk $\mathcal{P}_{6}$ (down figure). Calculation with L-SROM (red/grey line with crosses) and with NL-SROM2 (blue/black line with circles). 

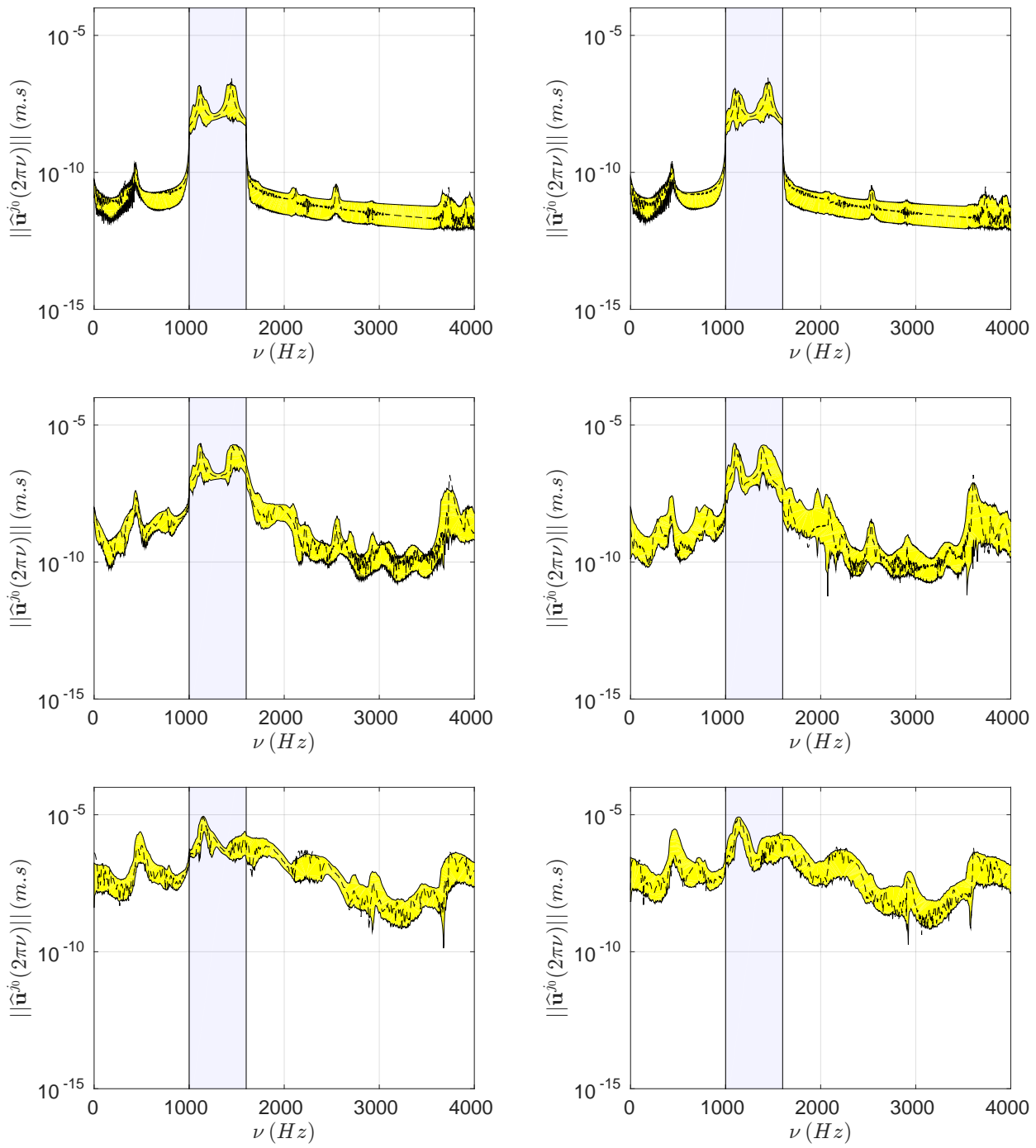

Figure 19: For $\delta_{K}=0.1$, confidence region (yellow/grey region) of $v \mapsto\left\|\widehat{\mathbf{U}}^{j_{0}}(2 \pi v)\right\|$ corresponding to a probability level 0.95 , computed using NL-SROM2 for $\mathcal{P}_{0}$ (left figures) and $\mathcal{P}_{6}$ (right figures), and for three values of parameter $s_{0}: s_{0}=0.01$ (top figures), $s_{0}=0.15$ (central figures), and $s_{0}=1$ (down figures). The dashed-line is the response of the deterministic mean (nominal) model. The vertical grey region corresponds to excitation frequency band $\mathbb{B}_{e}$ 


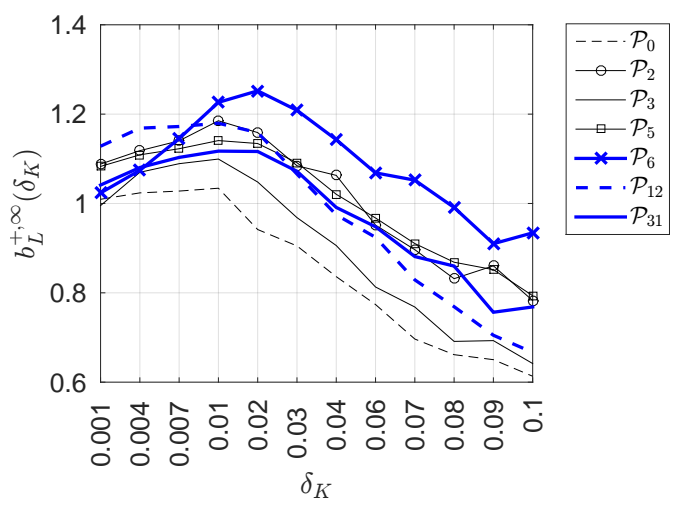

Figure 20: Graph of function $\delta_{K} \mapsto b_{\mathrm{L}}^{+, \infty}\left(\delta_{K}\right)$ for tuned pattern $\mathcal{P}_{0}$ and detuned ones $\mathcal{P}_{2}, \mathcal{P}_{3}, \mathcal{P}_{5}$, $\mathcal{P}_{6}, \mathcal{P}_{12}$, and $\mathcal{P}_{31}$ defined in Appendix A.
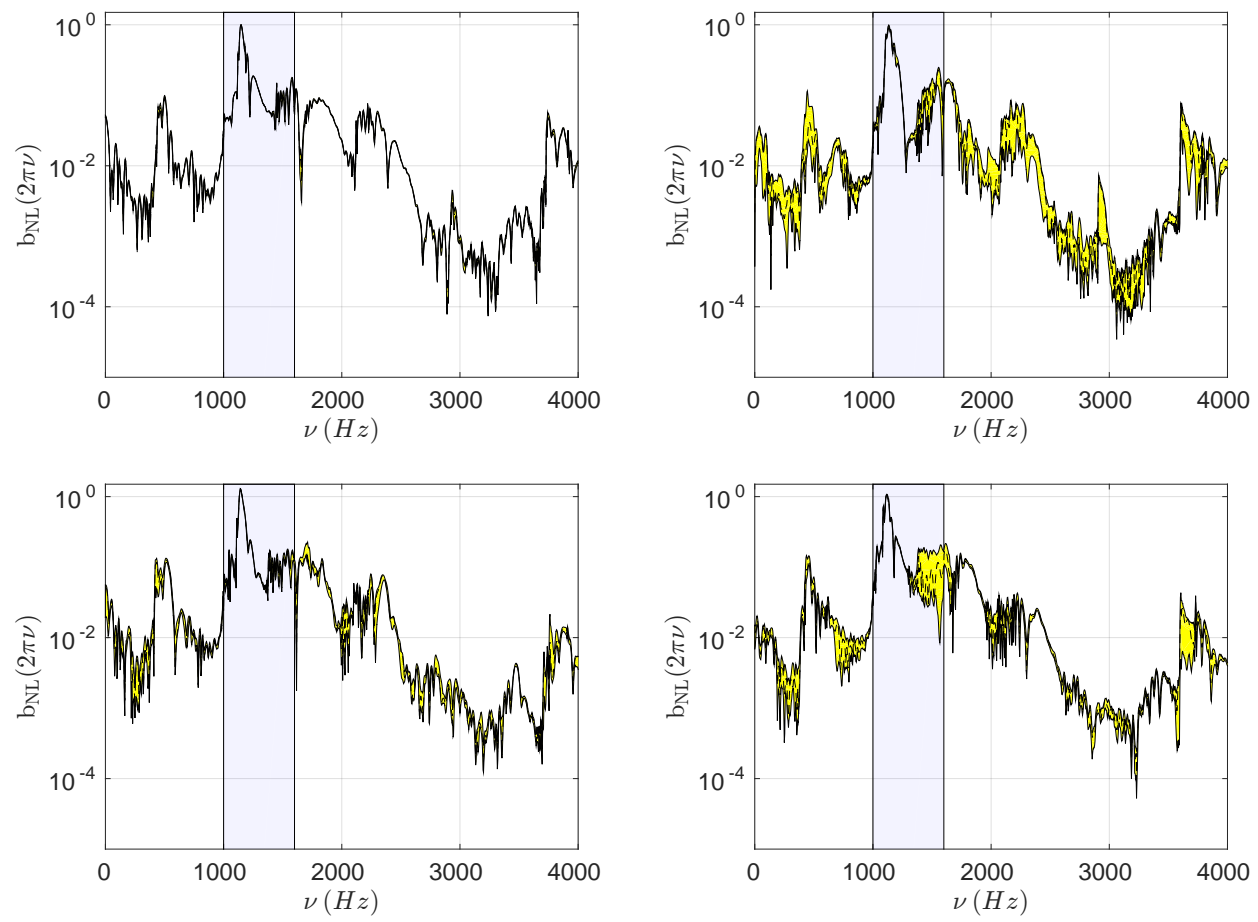

Figure 21: For $\delta_{K}=0.03$, confidence region (yellow/grey region) of the random amplification factor, $B_{\mathrm{NL}}(2 \pi v)$, estimated with a probability level of 0.95 using NL-SROM1, for the tuned configuration $\mathcal{P}_{0}$ (left top figure), and for the detuned configurations, $\mathcal{P}_{6}$ (right top), $\mathcal{P}_{11}$ (left down), and $\mathcal{P}_{25}$ (right down). The dashed-line is the nominal amplification factor $b_{\mathrm{NL}}(2 \pi v)$. The vertical grey region corresponds to excitation frequency band $\mathbb{B}_{e}$. 

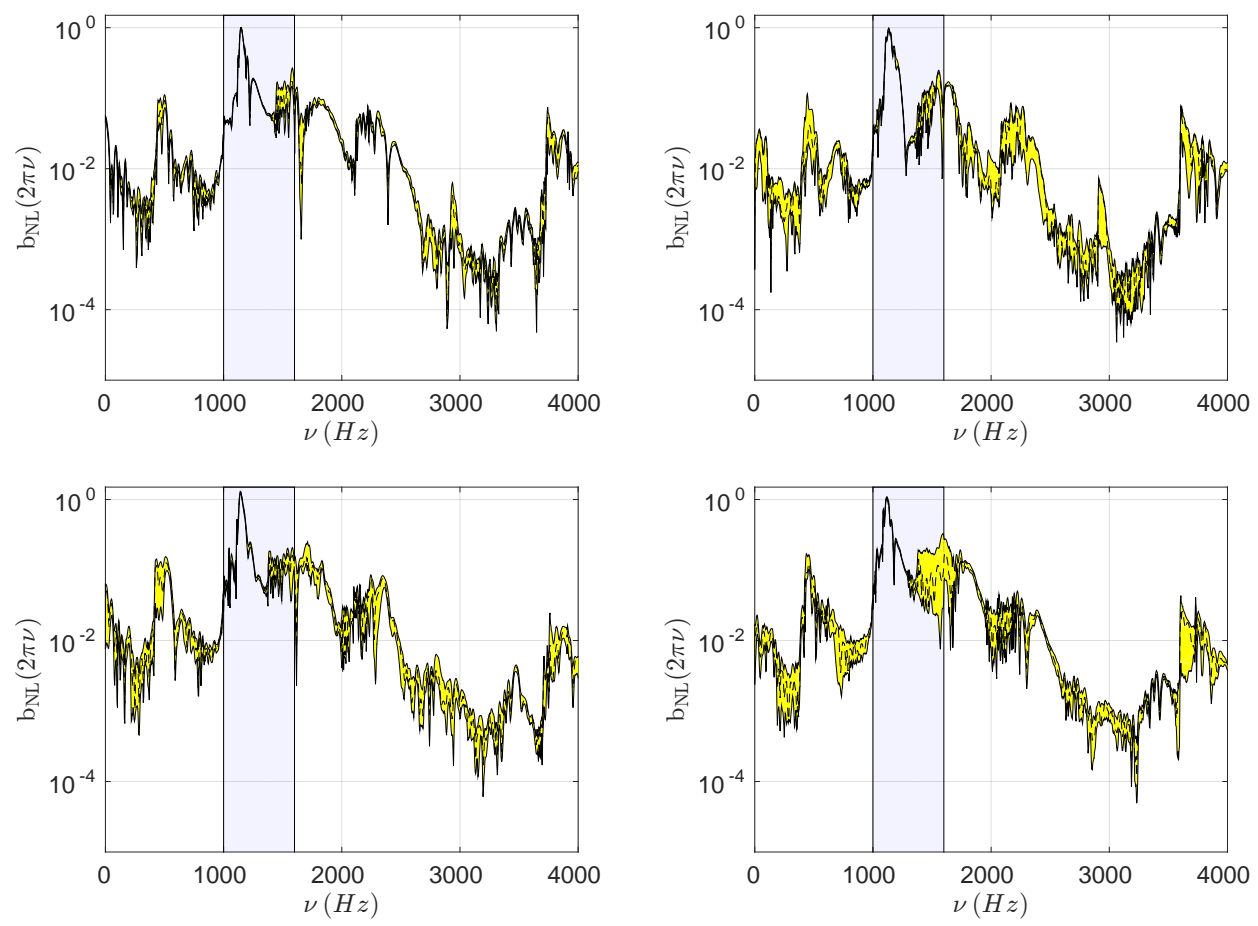

Figure 22: For $\delta_{K}=0.1$, confidence region (yellow/grey region) of the random amplification factor, $B_{\mathrm{NL}}(2 \pi v)$, estimated with a probability level of 0.95 using NL-SROM1, for the tuned configuration $\mathcal{P}_{0}$ (left top figure), and for the detuned configurations, $\mathcal{P}_{6}$ (right top), $\mathcal{P}_{11}$ (left down), and $\mathcal{P}_{25}$ (right down). The dashed-line is the nominal amplification factor $b_{\mathrm{NL}}(2 \pi v)$. The vertical grey region corresponds to excitation frequency band $\mathbb{B}_{e}$. 

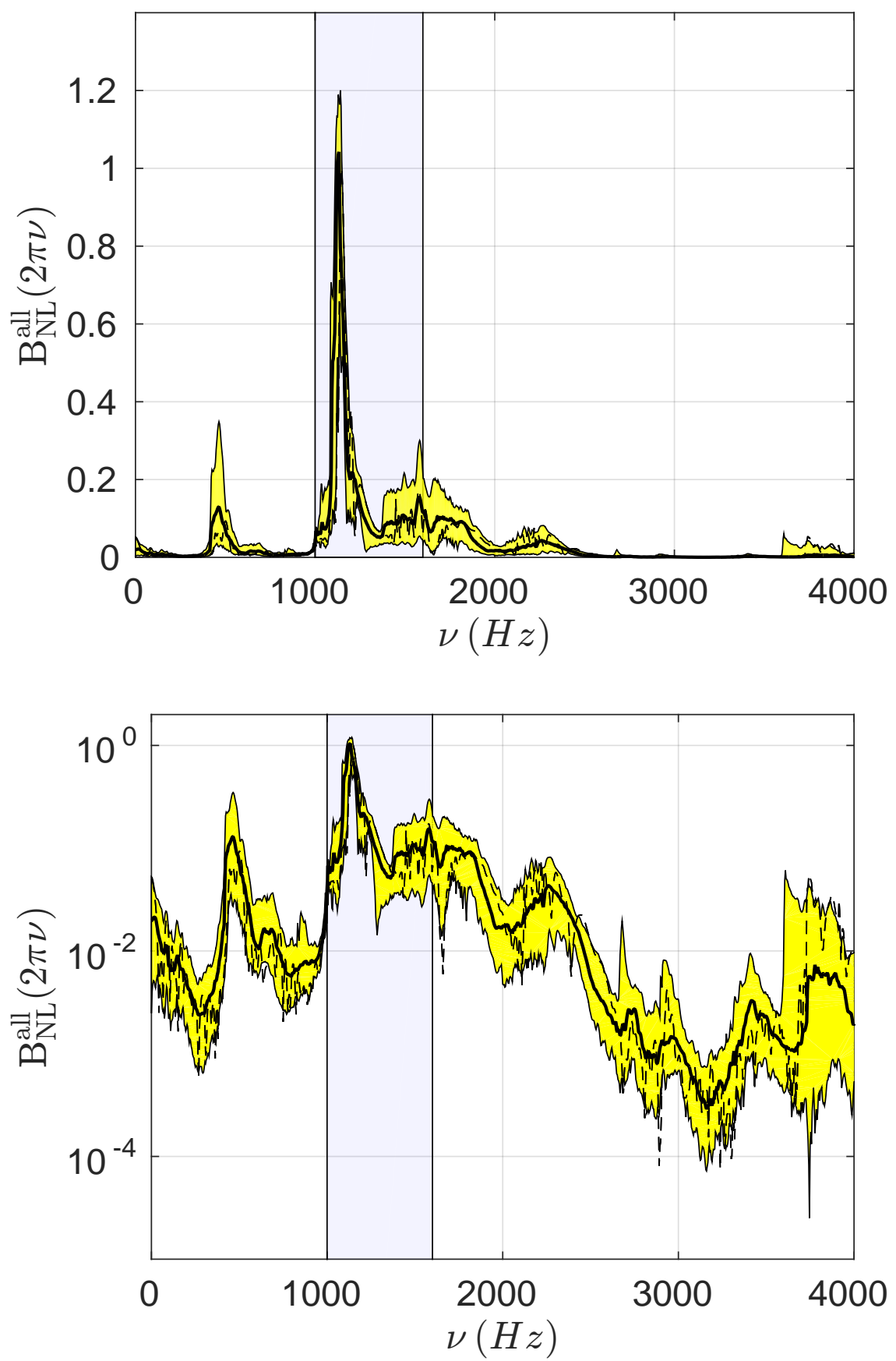

Figure 23: For $\delta_{K}=0.1$, confidence region (yellow/grey region) of the random amplification factor, $B_{\mathrm{NL}}^{\text {all }}(2 \pi v)$, related to the 46 patterns, estimated with a probability level of 0.95 using NLSROM1. The dashed-line is the amplification factor $b_{\mathrm{NL}}^{\text {tuned }}(2 \pi \nu)$ of the tuned system without mistuning. The thick solid line is the median B̧lue of random variable $B_{\mathrm{NL}}^{\text {all }}(2 \pi v)$. The vertical grey region corresponds to excitation frequency band $\mathbb{B}_{e}$. Linear scale (left figure), log scale (right figure) 


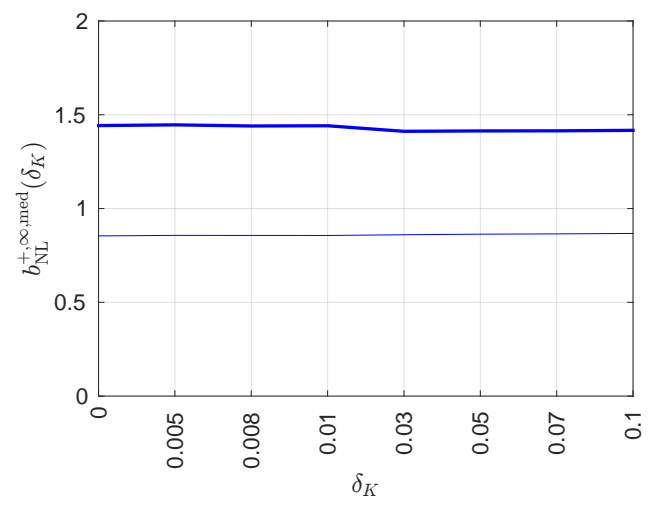

Figure 24: For band $\mathbb{B}_{\text {med }}$, lower (thin solid line) and upper (thick solid line) envelopes of the regions containing the 46 graphs of functions $\delta_{K} \mapsto b_{\mathrm{NL}}^{+, \infty, \text { med }}\left(\delta_{K}\right)$ for the 46 patterns. The upper envelope corresponds to pattern $\mathcal{P}_{33}$ and the lower one to $\mathcal{P}_{1}$.

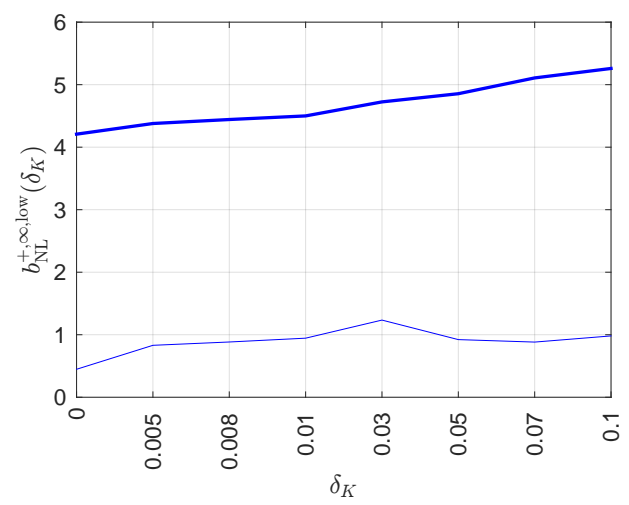

Figure 25: For band $\mathbb{B}_{\text {low }}$, lower (thin solid line) and upper (thick solid line) envelopes of the regions containing the 46 graphs of functions $\delta_{K} \mapsto b_{\mathrm{NL}}^{+, \infty \text { low }}\left(\delta_{K}\right)$ for the 46 patterns. The upper envelope corresponds to pattern $\mathcal{P}_{26}$ and the lower one to $\mathcal{P}_{37}$. 


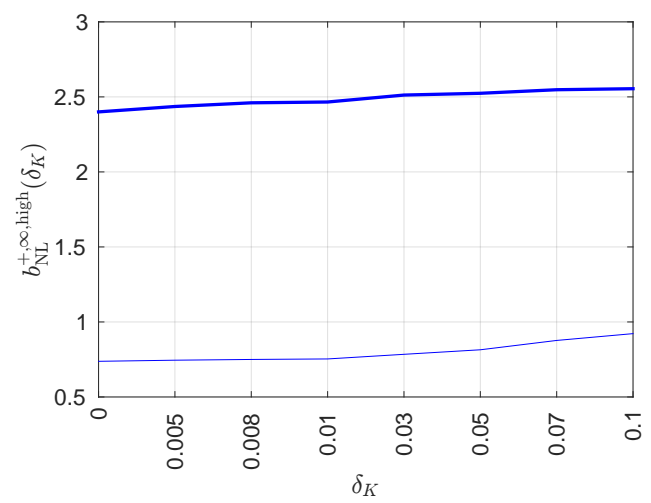

Figure 26: For band $\mathbb{B}_{\text {high }}$, lower (thin solid line) and upper (thick solid line) envelopes of the regions containing the 46 graphs of functions $\delta_{K} \mapsto b_{\mathrm{NL}}^{+, \infty \text { high }}\left(\delta_{K}\right)$ for the 46 patterns. The upper envelope corresponds to pattern $\mathcal{P}_{9}$ and the lower one to $\mathcal{P}_{8}$. 